\title{
Vanishing of cohomology with coefficients in representations on Banach spaces of groups acting on Buildings
}

\author{
Izhar Oppenheim \\ Department of Mathematics, Ben-Gurion University of the Negev, \\ Be'er Sheva 84105, Israel, izharo@bgu.ac.il
}

July 26, 2021

\begin{abstract}
We prove vanishing of cohomology with coefficients in representations on a large class of Banach spaces for a group acting "nicely" on a simplicial complexes based on spectral properties of the 1-dimensional links of the simplicial complex.
\end{abstract}

\section{Introduction}

The study of group actions on metric space is a broad topic in which one studies the interplay between the group structure and the structure of the metric space on which it acts. When considering a group action on Hilbert spaces, property $(\mathrm{FH})$ is an imporant notion which is defined as follows: a group $G$ has property (FH) if every isometric action of $G$ on a Hilbert space admits a fixed point. Property $(\mathrm{FH})$ can be rephrased in a cohomological language as follows: a group has property $(\mathrm{FH})$ if and only if $H^{1}(G, \pi)=0$ for any unitary representation of $G$ on a Hilbert space (the proof of this fact can be found for instance in [3] [Lemma 2.2.6]).

Recently there have been much interest in studying the generalization of property $(\mathrm{FH})$ for group actions on Banach spaces (see [16] for a survey of recent developments regarding this question). In order to state a generalization of property $(\mathrm{FH})$ in the Banach setting, we recall the following facts taken from [16] [Section 2.2]:

- Any affine action $A$ on a Banach space $X$ is of the form $A x=T x+b$ where $T$ is a linear map and $b \in X$.

- As a result of the previous fact, if $\rho$ defines an affine action of $G$ on $X$, then

$$
\forall g \in G, \rho(g) \cdot x=\pi(g) \cdot x+b(g),
$$

where $\pi: G \rightarrow B(X)$ is a linear representation of $G$ on $X$ called the linear part of $\rho$ and $b: G \rightarrow X$ is a map which satisfies the cocycle condition:

$$
\forall g, h \in G, b(g h)=\pi(g) b(h)+b(g) .
$$


- For a group $G$ and a linear representation $\pi$ on a Banach space $X$, $H^{1}(G, \pi)=0$ if and only if any affine action with a linear part $\pi$ admits a fixed point.

Thus the vanishing of the first cohomology reflects a rigidity phenomenon. In this article, we will explore a generalization of this phenomenon and we will prove vanishing of higher cohomologies for groups acting on simplicial complexes. The idea is that given a "nice enough" group action on a simplicial complex $\Sigma$, one can show vanishing of cohomology with coefficients in representations on Banach spaces under suitable assumption on the norm growth of the action and on the geometry of the simplicial complex. This is done by using the interplay between the geometry of the Banach space and the geometry of the simplicial complex as it is reflected in the angles between couples of subgroups of $G$ stabilizing top-dimensional simplices in the simplicial complex (see definition below).

The definition of angle between subgroups in the Hilbert setting is as follows: let $G$ be a group, $\pi$ by a unitary representation of $G$ on a Hilbert space $H$ and let $K_{1}, K_{2}<G$ be subgroups of $G$. The angle between $K_{1}$ and $K_{2}$ with respect to $\pi$ is defined as the (Friedrichs) angle between $H^{\pi\left(K_{1}\right)}$ and $H^{\pi\left(K_{2}\right)}$. The angle between $K_{1}$ and $K_{2}$ is then defined as the supremum with respect to all unitary representations of $G$.

This idea of angle was used in the work of Dymara and Januszkiewicz 8 to prove property $(\mathrm{T})$ (which is equivalent to property $(\mathrm{FH})$ in this setting) and vanishing of higher cohomologies with coefficients in representations on Hilbert spaces for groups acting on simplicial complexes. Dymara and Januszkiewicz further showed how to bound the angle between the two subgroups using the spectral gap of the Laplacian on a graph generated by these subgroups.

At first glance, this idea seems very much related to the so called "geometrization of property (T)" (this term was coined by Shalom 23), since it uses the spectral gap of a Laplacian to deduce property $(\mathrm{T})$ in a way similar to Zuk's famous criterion for property (T) (see [27, 2]). However, at its core, the idea of angle between subgroups is much stronger than Zuk's criterion, because it better captures the behaviour of the group $G$. In [17 the author generalized this idea of angle to the setting of Banach spaces, considering angle between projections instead of angle between subspaces. This new notion of angle was used by the author it to show a strengthened version of Banach property $(\mathrm{T})$ for a large class of Banach spaces. This in turn implies the vanishing of the first group cohomology with coefficients in the isometric representations on this class of Banach spaces.

The aim of this paper is to generalize the vanishing of cohomologies theorem of Dymara and Januszkiewicz in [8 to coefficients in representations on Banach spaces. A major problem with transfering the results of Dymara and Januszkiewicz to Banach space setting was that the angle computations of [8] [section 4] heavily relied on the idea that in Hilbert spaces the angle between two subspaces is equal to the angle between their orthogonal complements. However, this idea of computing the angle by passing to the orthogonal complement does not seem to work in our definition of angle between projections.

The technical heart of this paper is devoted to attaining results regarding angles between projections in Banach spaces that are similar to the results of Dymara and Januszkiewicz (but without passing to the orthogonal complemet). 
In order to attain these results, we first explore the idea of angle between more than two projections (this was inspired by the ideas of Kassabov in [13).

After obtaining these technical results, the vanishing theorem can be reproved for coefficients in representations on Banach spaces by the same arguments given in 8 .

In order to apply these results in concrete examples (such as groups groups coming from a BN-pair), we need to bound angles between pairs of subgroups $K_{1}, K_{2}<G$ with respect to representations on Banach spaces. Given a pair of subgroups $K_{1}, K_{2}<G$, this is done by bounding this angle between these subgroups in the Hilbert setting and then (if this angle is large enough to begin with) using this bound in order to get a bound on the angles between these subgroups with respect to representations on Banach spaces that are "close enough" to a Hilbert space. Being "close enough" to a Hilbert space involves a several step process of deforming a Hilbert space that will be explained in detail below.

\subsection{Deformations of Hilbert spaces}

We will consider Banach spaces which are deformations of Hilbert spaces. In order to explain which deformations we consider, we need to introduce several ideas from the theory of Banach spaces.

\subsubsection{The Banach-Mazur distance and Banach spaces with "round" subspaces}

The Banach-Mazur distance measures a distance between isomorphic Banach spaces:

Definition 1.1. Let $Y_{1}, Y_{2}$ be two isomorphic Banach spaces. The (multiplicative) Banach-Mazur distance between $Y_{1}$ and $Y_{2}$ is defined as

$$
d_{B M}\left(Y_{1}, Y_{2}\right)=\inf \left\{\|T\|\left\|T^{-1}\right\|: T: Y_{1} \rightarrow Y_{2} \text { is a linear isomorphism }\right\} .
$$

This distance has a multiplicative triangle inequality (the proof is left as an exercise to the reader):

Proposition 1.2. Let $Y_{1}, Y_{2}, Y_{3}$ be isomorphic Banach spaces. Then

$$
d_{B M}\left(Y_{1}, Y_{3}\right) \leq d_{B M}\left(Y_{1}, Y_{2}\right) d_{B M}\left(Y_{2}, Y_{3}\right) .
$$

We will be especially interested in the Banach-Mazur distance between $n$ dimensional Banach spaces and $\ell_{2}^{n}$. A classical theorem by F. John 12 states for every $n$-dimensional Banach space $Y, d_{B M}\left(Y, \ell_{2}^{n}\right) \leq \sqrt{n}$ and the classical cases in which this inequality is an equality are $\ell_{1}^{n}$ and $\ell_{\infty}^{n}$. Later, Milman and Wolfson [15] proved that these classical cases are in some sense generic: [15] [Theorem 1] states that if $d_{B M}\left(Y, \ell_{2}^{n}\right)=\sqrt{n}$, then there is $k \geq \frac{\ln (n)}{2 \ln (12)}$ such that $Y$ contains a $k$-dimensional subspace isometric to $\ell_{1}^{k}$.

In this paper we will concern ourselves with Banach spaces whose finite dimensional subspaces are sufficiently "round", i.e., sufficiently close to $\ell_{2}$-spaces. Given a Banach space $X$ and a constant $k \in \mathbb{N}$, we use the following notation $d_{k}(X)$ taken from the work of de Laat and de la Salle [7]:

$$
d_{k}(X)=\sup \left\{d_{B M}(Y): Y \subseteq X, \operatorname{dim}(Y) \leq k\right\} .
$$


We further introduce the following notation: given a constant $r \geq 2$ and a constant $C_{1} \geq 1$, we denote $\mathcal{E}_{1}\left(r, C_{1}\right)$ to be the class of Banach spaces defined as follows:

$$
\mathcal{E}_{1}\left(r, C_{1}\right)=\left\{X: \forall k \in \mathbb{N}, d_{k}(X) \leq C_{1} k^{\frac{1}{r}}\right\} .
$$

The reader should note that for every choice of $r \geq 2, C_{1} \geq 1$, the class $\mathcal{E}_{1}\left(r, C_{1}\right)$ always contains the class of all Hilbert spaces, since for every Hilbert space $H$ we have that $d_{k}(H)=1$ for every $k$.

An example of Banach spaces contained in $\mathcal{E}_{1}\left(r, C_{1}\right)$ are spaces of bounded type and cotype. The definitions of type and cotype are given in the background section below, but for our uses, it is sufficient to state the following theorem due to Tomczak-Jaegermann [24][Theorem 2 and the corollary after it]: if $X$ is a Banach space of type $p_{1}$, cotype $p_{2}$ and corresponding constants $T_{p_{1}}(X)$, $C_{p_{2}}(X)$ (see definitions below), then $d_{k}(X) \leq 4 T_{p_{1}}(X) C_{p_{2}}(X) k^{\frac{1}{p_{1}}-\frac{1}{p_{2}}}$.

This theorem yields that for every $r>2$, every $\frac{1}{r}$ and every $C_{1} \geq 1$, the class $\mathcal{E}_{1}\left(r, C_{1}\right)$ contains all Banach spaces $X$ with type $p_{1}$, cotype $p_{2}$ and corresponding constants $T_{p_{1}}(X), C_{p_{2}}(X)$ such that $\frac{1}{p_{1}}-\frac{1}{p_{2}} \leq \frac{1}{r}$ and $4 T_{p_{1}}(X) C_{p_{2}}(X) \leq C_{1}$.

\subsubsection{Interpolation}

Two Banach spaces $X_{0}, X_{1}$ form a compatible pair $\left(X_{0}, X_{1}\right)$ if there is a continuous linear embedding of both $X_{0}$ and $X_{1}$ in the same topological vector space. The idea of complex interpolation is that given a compatible pair $\left(X_{0}, X_{1}\right)$ and a constant $0<\theta<1$, there is a method to produce a new Banach space $\left[X_{0}, X_{1}\right]_{\theta}$ as a "combination" of $X_{0}$ and $X_{1}$. We will not review this method here, and the interested reader can find more information on interpolation in [5].

We will introduce the following notation: let $\mathcal{E}$ be a class of Banach spaces and let $0<\theta_{2} \leq 1$ be a constant. Denote $\mathcal{E}_{2}\left(\mathcal{E}, \theta_{2}\right)$ the class of Banach spaces defined as follows

$\mathcal{E}_{2}\left(\mathcal{E}, \theta_{2}\right)=\left\{X: \exists X_{1} \in \mathcal{E}, \exists X_{0}\right.$ Banach, $\exists \theta_{2} \leq \theta \leq 1$ such that $\left.X=\left[X_{0}, X_{1}\right]_{\theta}\right\}$

We will be interested with composing this definition with $\mathcal{E}_{1}\left(r, C_{1}\right)$ defined above and considering $\mathcal{E}_{2}\left(\mathcal{E}_{1}\left(r, C_{1}\right), \theta_{2}\right)$.

As noted above $\mathcal{E}_{1}\left(r, C_{1}\right)$ contains the class of all the Hilbert spaces. This brings us to consider the following definition is due to Pisier in 19: a Banach space $X$ is called strictly $\theta$-Hilbertian for $0<\theta \leq 1$, if there is a compatible pair $\left(X_{0}, X_{1}\right)$ such that $X_{1}$ is a Hilbert space such that $X=\left[X_{0}, X_{1}\right]_{\theta}$. Examples of strictly $\theta$-Hilbertian spaces are $L^{p}$ space and non-commutative $L^{p}$ spaces, where in these cases $\theta=\frac{2}{p}$ if $2 \leq p<\infty$ and $\theta=2-\frac{2}{p}$ if $1<p \leq 2$ (a reader who is not familiar with non-commutative $L^{p}$ spaces can find a detailed account in [21]).

Another source of examples for strictly $\theta$-Hilbertian spaces are superreflexive Banach lattices. Recall that a Banach space $X$ is called uniformly convex if

$$
\sup \left\{\left\|\frac{x+y}{2}\right\|: \forall x, y \in X,\|x\|=\|y\|=1,\|x-y\| \geq \varepsilon\right\}<1 \text { for every } \varepsilon>0 .
$$

Further recall that a Banach space $X$ is called superreflexive if all its ultrapowers are reflexive, which is equivalent by [4][Theorem A.6] to $X$ being isomorphic to 
a uniformly convex space. A Banach lattice is a Banach space with a "wellbehaved" partial order on it - the definition is rather techincal and we will not recall it here (for the exact defition of a Banach lattice and further properties of it, the reader is reffered to [4] $[$ Appendix G]).

Pisier [19] proved that any superreflexive Banach lattice is strictly $\theta$-Hilbertian and suggested that this result might by true even for superreflexive Banach spaces which are not Banach lattices.

\subsubsection{Passing to a isomorphic space}

The last deformation we want to consider is passing to an isomorphic space. We introduce the following notation: let $\mathcal{E}$ be a class of Banach spaces and let $C_{3} \geq 1$ be a constant, denote by $\mathcal{E}_{3}\left(\mathcal{E}, C_{3}\right)$ the class of Banach spaces defined as

$$
\mathcal{E}_{3}\left(\mathcal{E}, C_{3}\right)=\left\{X: \exists X^{\prime} \in \mathcal{E} \text { such that } d_{B M}\left(X, X^{\prime}\right) \leq C_{3}\right\} .
$$

\subsubsection{Passing to the closure}

Our criterion for vanishing of cohomology relies on geometric properties of a Banach space that are stable under certain operations. Therefore, we can enlarge our Banach class by passing to the closure under these operations: for a class of Banach spaces $\mathcal{E}$, denote by $\overline{\mathcal{E}}$ the smallest class of Banach spaces containing $\mathcal{E}$ that is closed under passing to quotients, subspaces, $l_{2}$-sums and ultraproducts.

\subsubsection{Composing the deformations}

The class of Banach spaces we will want to consider is the composition of the all the deformations described above, i.e., we start with a Hilbert space and use $\mathcal{E}_{1}\left(r, C_{1}\right)$ to consider deformations of it, on that class we consider interpolation, then pass to isomorphic spaces with bounded Banach-Mazur distance and finish by passing to the closure. To put it all together, we start with constants $r \geq 2$, $C_{1} \geq 1,1 \geq \theta_{2}>0$ and $C_{3} \geq 1$ and consider the class $\overline{\mathcal{E}_{3}\left(\mathcal{E}_{2}\left(\mathcal{E}_{1}\left(r, C_{1}\right), \theta_{2}\right), C_{3}\right)}$.

\subsection{The main theorem for BN-pair groups}

Following Dymara and Januszkiewicz, our vanishing of cohomology results are true for groups acting on simplicial complexes given that certain conditions are fulfilled (conditions $(\mathcal{B} 1)-(\mathcal{B} 4)$ and $\left(\mathcal{B}_{\delta, r}\right)$ stated below). However, currently, our only examples of groups acting on complexes satisfying these conditions are groups with a BN-pair (e.g., classical BN-pair groups acting on Euclidean buildings or 2-spherical Kac-Moody groups). Therefore, in this introduction we will state our main result only for BN-pair groups (the more general Theorem 4.7 is given below).

In order to state the main theorem, we recall some generalities regarding $\mathrm{BN}$-pair groups (a reader not familiar with BN-pair groups can find and extensive treatment of this subject in [1] [Chapter 6]) and introduce a few notations regarding representations.

Let $G$ be a BN-pair group and let $\Sigma$ be the $n$-dimensional building on which it acts. Then $G$ acts on $\Sigma$ cocompactly and $\triangle=\Sigma / G$ is a single chamber of $\Sigma$. We assume that $n>1$, i.e., that $\Sigma$ is not a tree and denote $\triangle(k)$ to be the $k$-dimensional faces of $\Sigma / G$. We assume further that there is some $l \in \mathbb{N}$ 
such that all the $l$-dimensional links of $\Sigma$ are compact. Be this assumption, for every $\tau \in \triangle(n-2)$, the isotropy group $G_{\tau}=\operatorname{Stab}(\tau)$ is compact and $G$ is generated by $\bigcup_{\tau \in \triangle(n-2)} G_{\tau}$. Let $\mathcal{E}$ be a class of Banach spaces and let $s_{0}>0$ be a constant. Denote $\mathcal{F}\left(\mathcal{E}, G, s_{0}\right)$ to be all the continuous representations $(\pi, X)$ of $G$ such that $X \in \mathcal{E}$ and

$$
\sup _{g \in \bigcup_{\tau \in \Delta(n-2)} G_{\tau}}\|\pi(g)\| \leq e^{s_{0}} .
$$

Note that $\mathcal{F}\left(\mathcal{E}, G, s_{0}\right)$ contains all the isometric representation of $G$ on some $X \in \mathcal{E}$, but is also contains representations which are not uniformly bounded. Indeed, if $G$ is taken with the word norm |.| with respect to $\bigcup_{\tau \in \triangle(n-2)} G_{\tau}$, then $\mathcal{F}\left(\mathcal{E}, G, s_{0}\right)$ contains all the representations $\pi$ such that $\|\pi(g)\| \leq e^{s_{0}|g|}$ for every $g \in G$. Denote further $\mathcal{F}_{0}\left(\mathcal{E}, G, s_{0}\right)$ to be

$$
\mathcal{F}_{0}\left(\mathcal{E}, G, s_{0}\right)=\left\{\pi \in \mathcal{F}\left(\mathcal{E}, G, s_{0}\right): \pi^{*} \text { is a continuous representation }\right\},
$$

where $\pi^{*}$ is the dual representation of $\pi$.

After all these notations and definitions, we are ready to state our main theorem:

Theorem. Let $G$ be a group coming from a BN-pair and let $\Sigma$ be the $n$ dimensional building on which it acts. Assume that $n>1$ and there is some $l \in \mathbb{N}$ such that all the l-dimensional links of $\Sigma$ are compact. Denote by $q+1$ the thickness of the building $\Sigma$.

Let $r>20, C_{1} \geq 1,1 \geq \theta_{2}>0, C_{3} \geq 1$ be constants. Then there are constants $s_{0}=s_{0}(n)$ and $Q=Q\left(n, C_{1}, \theta_{2}, C_{3}\right)$ such that if $q \geq Q$, then for every

$$
\pi \in \mathcal{F}_{0}\left(\overline{\mathcal{E}_{3}\left(\mathcal{E}_{2}\left(\mathcal{E}_{1}\left(r, C_{1}\right), \theta_{2}\right), C_{3}\right)}, G, s_{0}\right),
$$

we have that

$$
H^{i}(G, \pi)=0, i=1, \ldots, l .
$$

Remark 1.3. In the above theorem, when considering $\mathcal{E}_{1}\left(r, C_{1}\right)$ we took $r>20$. In many cases, this choice can be improved, i.e., $r$ can be taken to be smaller, if the codimension 1 links of the building $\Sigma$ are known. For instance, if $\Sigma$ is known to be an $\widetilde{A}_{n}$ building, then we have the same theorem above with $r>4$. The precise statement of this fact is given in Corollary 4.8

\subsection{Examples of Banach spaces for which the theorem holds}

In the main theorem above, we considered only representations whose dual is continuous. This might seem to be a major restriction, but we will show below that the class of representations that we are considering is still very rich. We will do so by showing that there are interesting examples (families of) of Banach spaces in $\overline{\mathcal{E}_{3}\left(\mathcal{E}_{2}\left(\mathcal{E}_{1}\left(r, C_{1}\right), \theta_{2}\right), C_{3}\right)}$ with $r>20$ for which each continuous representation has a continuous dual.

Indeed, 14][Corollary 6.9] states that if $X$ is an Asplund Banach space then for every continuous representation $\pi$, the dual representation $\pi^{*}$ is also continuous. The exact definition of Asplund spaces in given in the next section (along with a good reference regarding these spaces), but for our needs, it is 
enough to recall that any reflexive Banach space is an Asplund space. Using this fact, we will show that $\overline{\mathcal{E}_{3}\left(\mathcal{E}_{2}\left(\mathcal{E}_{1}\left(r, C_{1}\right), \theta_{2}\right), C_{3}\right)}$ contains many interesting reflexive spaces.

First, for a Banach space $X$, we recall that $X$ is called uniformly non-square if there is some $\varepsilon>0$ such that for every $x, y \in X$ with in the unit ball of $X, \min \left\{\left\|\frac{x+y}{2}\right\|,\left\|\frac{x-y}{2}\right\|\right\} \leq 1-\varepsilon$. James [11][Theorem 1.1] showed that every uniformly non-square space is reflexive. An easy exercise shows that if $d_{2}(X)<$ $\sqrt{2}$ then $X$ is uniformly non-square. Therefore, for every $X \in \mathcal{E}_{1}\left(r, C_{1}\right)$, if $d_{2}(X)<\sqrt{2}$, then $X$ is reflexive, i.e., every $X \in \mathcal{E}_{1}\left(r, C_{1}\right)$ whose 2-dimensional subspaces are not too distorted is a reflexive space.

Second, since $\mathcal{E}_{1}\left(r, C_{1}\right)$ contains all Hilbert space, we have that $\mathcal{E}_{2}\left(\mathcal{E}_{1}\left(r, C_{1}\right), \theta_{2}\right)$ contains all $\theta$-Hilbertian spaces with $\theta \geq \theta_{2}$. As noted above this includes $L^{p}$ spaces and non commutative $L^{p}$ spaces with $\frac{2}{2-\theta_{2}} \leq p \leq \frac{2}{\theta_{2}}$. By [21][Theorem 5.1] these spaces are uniformly convex and therefore superreflexive (hence reflexive). Also, $\mathcal{E}_{2}\left(\mathcal{E}_{1}\left(r, C_{1}\right), \theta_{2}\right)$ also includes a subclass of the class of superreflexive Banach lattices (as noted above, for any superreflexive Banach lattice $X$, there is $\theta>0$ such that $X$ is $\theta$-Hilbertian).

Third, reflexivity of Banach spaces is preserved under isomorphism and therefore $\mathcal{E}_{3}\left(\mathcal{E}_{2}\left(\mathcal{E}_{1}\left(r, C_{1}\right), \theta_{2}\right), C_{2}\right)$ contains isomorphic spaces to the reflexive Banach spaces contained in $\mathcal{E}_{2}\left(\mathcal{E}_{1}\left(r, C_{1}\right), \theta_{2}\right)$.

Last, reflexivity is preserved under passing to a closed subspace, taking a quotient by a closed subspace and countable $l_{2}$-sums. Ultrapowers does not preserve reflexivity, but by definition, if $X$ is superreflexive, then all its ultrapowers are reflexive. Therefore passing to the closure $\overline{\mathcal{E}_{3}\left(\mathcal{E}_{2}\left(\mathcal{E}_{1}\left(r, C_{1}\right), \theta_{2}\right), C_{3}\right)}$ provides more examples of reflexive Banach spaces constructed from $\mathcal{E}_{3}\left(\mathcal{E}_{2}\left(\mathcal{E}_{1}\left(r, C_{1}\right), \theta_{2}\right), C_{3}\right)$ by these operations.

Remark 1.4. Above we have a list of families of Banach spaces (e.g., $L^{p}$ spaces or uniformly non-square spaces in $\mathcal{E}\left(r, C_{1}\right)$ when $\left.r>20\right)$ for which our main theorem holds for every representation in which the norm doesn't grow too fast with respect to the word norm (in particular, for every isometric representation). As far as we know, for each one of these families our vanishing of higher cohomologies results are new even in the classical case of BN-pair groups acting on Euclidean buildings.

Structure of this paper. Section 2 includes all the needed background material. Section 3 is devoted to proving the main technical result regarding angles between projections in Banach spaces. In section 4, we formulate and prove our main results regarding vanishing of cohomologies for groups acting on simplicial complexes. The appendix contains technical results regarding angles between projections under a weaker assumptions that the ones used in section 3 , that may be of independent interest.

\section{Background}

\subsection{Groups acting on simplicial complexes}

Here we present the set up needed for our results of groups acting on simplicial complexes. We start by recalling some definitions given by Dymara and Januszkiewicz in [8] [section 1]. 
Let $\Sigma$ be a countable pure $n$-dimensional simplicial complex with $n \geq 2$. The top dimensional simplices of $\Sigma$ will be called chambers and $\Sigma$ will be called gallery connected if for any two chambers $\sigma, \sigma^{\prime}$ there is a sequence of chambers

$$
\sigma=\sigma_{1}, \sigma_{2}, \ldots, \sigma_{k}=\sigma^{\prime}
$$

such that for every $i, \sigma_{i} \cap \sigma_{i+1}$ is a simplex of co-dimension 1 in $\Sigma$.

Denote by $A u t(\Sigma)$ the group of simplicial automorphisms of $\Sigma$. On $\operatorname{Aut}(\Sigma)$ define the compact-open topology whose basis are the sets $U\left(K, g_{0}\right)$ where $g_{0} \in$ $\operatorname{Aut}(\Sigma), K \subseteq \Sigma$ compact and $U\left(K, g_{0}\right)$ is defined as

$$
U\left(K, g_{0}\right)=\left\{g \in \operatorname{Aut}(\Sigma):\left.g\right|_{K}=\left.g_{0}\right|_{K}\right\} .
$$

Let $G<A u t(\Sigma)$ be a closed subgroup of $A u t(\Sigma)$.

Given a continuous representation $\pi$ of $G$ on a Banach space, one can define $H^{*}(G, \pi)$ and $H^{*}(\Sigma, \pi)$. We will not review these definitions here and a reader unfamilier with these definitions can find them in 8][Section 3] and reference therein. The main fact that we will use is that one can compute $H^{*}(G, \pi)$ based on $H^{*}(\Sigma, \pi)$ :

Lemma 2.1. [6][X.1.12] Let $\Sigma$ be a simplicial complex, $G<$ Aut $(\Sigma)$ be a closed subgroup and $\pi$ be a representation of $G$ on a Banach space. Assume that $\Sigma$ is contractible and locally finite and that the action of $G$ on $\Sigma$ is cocompact, then $H^{*}(G, \pi)=H^{*}(\Sigma, \pi)$

The above lemma assumes that $\Sigma$ is locally finite (i.e., that the link of every vertex is compact). In order to compute the cohomology of $G$ in cases where $\Sigma$ is not locally finite, Dymara and Januszkiewicz introduced the following definition of the core of $\Sigma$ :

Definition 2.2. 8][Definition 1.3] Let $\Sigma$ be a simplicial complex such that every link of $\Sigma$ is either compact or contractible (including $\Sigma$ itself, which is the link of the empty set) and such that the 0-dimensional links of $\Sigma$ are finite. Denote $\Sigma^{\prime}$ to be the first barycentric subdivision of $\Sigma$. The core of $\Sigma$, denoted $\Sigma_{D}$, is the subcomplex of $\Sigma^{\prime}$ spanned by the barycenters of simplices of $\Sigma$ with compact links.

Lemma 2.3. 8][Lemma 1.4] Let $\Sigma$ be an infinite simplicial complex such that every link of $\Sigma$ is either compact or contractible (in particular $\Sigma$ is contractible, because it is the link of $\emptyset$ ) and such that the 0 -dimensional links of $\Sigma$ are finite. Then $\Sigma_{D}$ is contractible.

Note that if the assumption that the 0-dimensional links of $\Sigma$ are finite implies that $\Sigma_{D}$ is locally finite. Also note that any closed subgroup $G<\operatorname{Aut}(\Sigma)$ is also a closed subgroup in $\operatorname{Aut}\left(\Sigma_{D}\right)$. Therefore combining the above lemma with Lemma 2.1 above yields the following corollary:

Corollary 2.4. Let $\Sigma$ be an infinite pure $n$-dimensional simplicial complex, $G<\operatorname{Aut}(\Sigma)$ be a closed subgroup and $\pi$ be a representation of $G$ on a Banach space. Assume that every link of $\Sigma$ is either compact or contractible and such that the 0-dimensional links of $\Sigma$ are finite. If the action of $G$ on $\Sigma$ is cocompact, then $H^{*}(G, \pi)=H^{*}\left(\Sigma_{D}, \pi\right)$. 
Following Dymara and Januszkiewicz, we will use the above corollary to show vanishing of the group cohomology under additional assumptions on $\Sigma$ and on the action of $G$. In order to state our additional assumptions we recall the following conditions on the couple $(\Sigma, G)$ taken from [8]:

(B1) All the 0-dimensional links are finite.

$(\mathcal{B} 2)$ All the links of dimension $\geq 1$ are gallery connected.

$(\mathcal{B} 3)$ All the links are either compact or contractable (including $\Sigma$ itself).

(B4) $G$ acts transitively on chambers and $\Sigma \rightarrow \Sigma / G$ restricts to an isomorphism on every chamber.

Let $\Sigma$ be an infinite simplicial complex and $G<A u t(\Sigma)$ be a closed subgroup satisfying $(\mathcal{B} 1)-(\mathcal{B} 4)$ and let $\pi$ a continuous representation of $G$ on a Banach space $X$. Fix a chamber $\triangle \in \Sigma(n)$ and for every $\eta \subseteq \triangle$, denote $G_{\eta}$ to be the subgroup of $G$ fixing $\sigma$ and also denote $X^{\pi\left(G_{\eta}\right)}=X_{\eta}$ to be the subspace of $X$ fixed by $G_{\eta}$ (under the action of $\pi$ ). One of the key ideas in [8] is that one can deduce vanishing of cohomologies of $G$ with coefficients in $\pi$ given that there are projections on all the $X_{\eta}$ 's and nice decompositions of these $X_{\eta}$ 's with respect to these projections. To make this precise:

Theorem 2.5. [8][Theorems 5.2,7.1] Let $\Sigma$ be an infinite simplicial complex, $G<\operatorname{Aut}(\Sigma)$ be a closed subgroup satisfying $(\mathcal{B} 1)-(\mathcal{B} 4)$ and $\pi$ a continuous representation of $G$ on a Banach space $X$. Under the notations above, for every $\eta \subseteq \triangle$ denote $D_{\eta}$ to be the subcomplex of $\Sigma_{D}$ spanned by the barycenters of simplices of $\triangle$ that have compact links and do not contain $\eta$.

Assume that for every $\eta \subseteq \triangle$ there is a projection $P_{\eta}: X \rightarrow X$ on $X_{\eta}$. For every $\eta \subseteq \triangle$, denote

$$
X^{\eta}=\operatorname{Im}\left(P_{\eta}\right) \cap \bigcap_{\tau \varsubsetneqq \eta} \operatorname{Ker}\left(P_{\tau}\right) .
$$

If for every $\eta \subseteq \triangle$, the following holds

$$
X_{\eta}=\bigoplus_{\tau \subseteq \eta} X^{\tau}
$$

then

$$
H^{*}(G, \pi)=\bigoplus_{\eta \subseteq \triangle} \widetilde{H}^{*-1}\left(D_{\eta} ; X^{\eta}\right)
$$

Moreover, if there is $l \geq 1$ such that all the $l$-dimensional links of $\Sigma$ are compact, then for every $i=1, \ldots, l, H^{i}(G, \pi)=0$.

Remark 2.6. In [8][Theorem 7.1] the assumptions of the theorem do not include the decomposition $X_{\eta}=\bigoplus_{\tau \subseteq \eta} X^{\tau}$, but assumptions regrading the spectral gap in the 1-dimensional links from which this decomposition is deduced. However, the proof of the theorem only relies on the above decomposition, therefore the theorem can be stated as above. Also, [8][Theorems 5.2, 7.1] are stated for continuous unitary representations on Hilbert spaces, but the proof of [8][Theorem 7.1] and the proof of [8][Theorem 5.2] based on [8][Theorem 7.1] pass verbatim to continuous representations on Banach spaces. 
We would like to add an additional condition on $\Sigma$ that will be denoted $\left(\mathcal{B}_{\delta, r}\right)$ (replacing the condition $\left(\mathcal{B}_{\delta}\right)$ appearing in $[8]$ ):

$\left(\mathcal{B}_{\delta, r}\right)$ For every $\eta \in \Sigma(n-2)$, the link of $\eta$, denoted $\Sigma_{\eta}$, is finite bipartite graph with sides $V_{\eta, 1}, V_{\eta, 2}$. For any $\eta \in \Sigma(n-2)$ denote

$$
V_{\text {min }}(\eta)=\min \left\{\left|V_{\eta, 1}\right|,\left|V_{\eta, 2}\right|\right\},
$$

and denote $\kappa(\eta)$ to be the smallest positive eigenvalue of the normalized Laplacian of $\Sigma_{\eta}$, then

$$
(1-\kappa(\eta))\left(V_{\min }(\eta)\right)^{\frac{1}{r}} \leq \delta .
$$

Remark 2.7. We note that if condition $(\mathcal{B} 4)$ is fulfilled and if the 1-dimensional links of $\Sigma$ are finite, then every 1-dimensional link has to be a bipartite graph.

The main source of examples of $(\Sigma, G)$ fulfilling $(\mathcal{B} 1)-(\mathcal{B} 4)$ and $\left(\mathcal{B}_{\delta, r}\right)$ are groups coming from BN-pairs (a reader unfamilier with the definition of a BNpair can find it in [1] [Chapter 6]), when $G$ is the group and $\Sigma$ is the building on which it acts. In 8 the following is proved:

Proposition 2.8. [8][Propositions 1.6,1.7] Let $G$ be a group coming from a $B N$-pair and let $\Sigma$ be the building on which it acts. Assume further that $\Sigma$ is non compact and has finite thickness. Then conditions $(\mathcal{B} 1)-(\mathcal{B} 4)$ are fulfilled for $(\Sigma, G)$ and $\Sigma_{D}$ is contractible.

In order to check the condition $\left(\mathcal{B}_{\delta, r}\right)$ in buildings, we recall that if a building $\Sigma$ has finite 1-dimensional links, then these links are spherical building, i.e., they are thick generalized $m$-gons with $m=2,3,4,6,8$ (a reader unfamilier with generalized $m$-gons can find a good introduction in 25] [Chapter 1]).

Proposition 2.9. Let $\Sigma$ be a building such that the 1-dimensional links of $\Sigma$ are compact. Let $m^{\prime}$ be the smallest integer such that all the links of 1-dimensional links of $\Sigma$ are generalized $m$-gons with $m \leq m^{\prime}$. Then for every

$$
r> \begin{cases}4 & m^{\prime}=3 \\ 8 & m^{\prime}=4 \\ 18 & m^{\prime}=6 \\ 20 & m^{\prime}=8\end{cases}
$$

and every $\delta>0$, if the thickness of the building is large enough, then $\left(\mathcal{B}_{\delta, r}\right)$ holds for $\Sigma$.

Proof. Let $(V, E)$ be a generalized $m$-gon of order $(s, t)$ and assume without loss of generality that $s \geq t$. Denote $\kappa$ to be the smallest positive eigenvalue of the normalized Laplacian on $(V, E)$. If $m=2$, then $1-\kappa=0$ and therefore this case is of no interest to us.

For $m>2$ the spectral gap $\kappa$ was explicitly computed by Feit and Higman 10] for all generalized $m$-gons (the reader can find a summation of these results in [2] [Section 3]). We will not recall the exact values of $\kappa$ depending on $(s, t)$, but only the asymptotic behaviour of $1-\kappa$ as $s$ and $t$ tends to $\infty$ :

$$
1-\kappa \sim\left\{\begin{array}{ll}
O\left(\frac{1}{\sqrt{t}}\right)=O\left(\frac{1}{\sqrt{s}}\right) & m=3 \\
O\left(\frac{1}{\sqrt{t}}+\frac{1}{\sqrt{s}}\right) & m=4,6,8
\end{array} .\right.
$$


We recall that generalized $m$-gons are always bipartite graphs. Denote $V_{1}, V_{2}$ to be the vertices in the two sides of $(V, E)$ and denote

$$
V_{\min }=\min \left\{\left|V_{1}\right|,\left|V_{2}\right|\right\} \text {. }
$$

The exact value of $\left|V_{\min }\right|$ depending on $(s, t)$ is computed in 25] [Corollary 1.5.5] (recall we assumed that $s \geq t$ ):

$$
V_{\text {min }}=\left\{\begin{array}{ll}
t^{2}+t+1 & m=3 \\
(s t+1)(t+1) & m=4 \\
\left.\left((s t)^{2}+s t+1\right)\right)(t+1) & m=6 \\
\left.\left((s t)^{2}+1\right)(s t+1)\right)(t+1) & m=8
\end{array} .\right.
$$

In order to complete the proof, we will also need the following connections between $s$ and $t$ (see [25] [Theorem 1.7.2]):

$$
\begin{cases}s=t & m=3 \\ t^{\frac{1}{2}} \leq s \leq t^{2} & m=4,8 . \\ t^{\frac{1}{3}} \leq s \leq t^{3} & m=6\end{cases}
$$

To conclude the proof, we combine all of the above in order to show that for $r$ as above, $(1-\kappa)\left|V_{\min }\right|^{\frac{1}{r}}$ tends to 0 as $t$ tends to infinity.

$$
\begin{aligned}
& (1-\kappa)\left|V_{\min }\right|^{\frac{1}{r}} \sim \begin{cases}\left(t^{2}+t+1\right)^{\frac{1}{r}} \frac{1}{\sqrt{t}} & m=3 \\
(s t+1)^{\frac{1}{r}}(t+1)^{\frac{1}{r}}\left(\frac{1}{\sqrt{t}}+\frac{1}{\sqrt{s}}\right) & m=4 \\
\left.\left((s t)^{2}+s t+1\right)\right)^{\frac{1}{r}}(t+1)^{\frac{1}{r}}\left(\frac{1}{\sqrt{t}}+\frac{1}{\sqrt{s}}\right) & m=6 \\
\left.\left((s t)^{2}+1\right)(s t+1)\right)^{\frac{1}{r}}(t+1)^{\frac{1}{r}}\left(\frac{1}{\sqrt{t}}+\frac{1}{\sqrt{s}}\right) & m=8\end{cases} \\
& \left\{\begin{array}{ll}
\left(t^{2}+t+1\right)^{\frac{1}{r}} \frac{1}{\sqrt{t}} & m=3 \\
\left(t^{2} t+1\right)^{\frac{1}{r}}(t+1)^{\frac{1}{r}}\left(\frac{1}{\sqrt{t}}+\frac{1}{\sqrt{t}}\right) & m=4 \\
\left.\left(\left(t^{3} t\right)^{2}+t^{3} t+1\right)\right)^{\frac{1}{r}}(t+1)^{\frac{1}{r}}\left(\frac{1}{\sqrt{t}}+\frac{1}{\sqrt{t}}\right) & m=6 \\
\left.\left(\left(t^{2} t\right)^{2}+1\right)\left(t^{2} t+1\right)\right)^{\frac{1}{r}}(t+1)^{\frac{1}{r}}\left(\frac{1}{\sqrt{t}}+\frac{1}{\sqrt{t}}\right) & m=8
\end{array} \leq\left\{\begin{array}{ll}
3^{\frac{1}{r}} t^{\frac{2}{r}} \frac{1}{\sqrt{t}} & m=3 \\
4^{\frac{1}{r}} t^{\frac{4}{r}} \frac{2}{\sqrt{t}} & m=4 \\
6^{\frac{1}{r}} t^{\frac{9}{r}} \frac{2}{\sqrt{t}} & m=6 \\
8^{\frac{1}{r}} t^{\frac{10}{r}} \frac{2}{\sqrt{t}} & m=8
\end{array},\right.\right.
\end{aligned}
$$

and the conclusion follows.

\subsection{Averaged projections in a Banach space}

Let $X$ be a Banach space. Recall that a projection $P$ is a bounded operator $P \in \mathcal{B}(X)$ such $P^{2}=P$. Note that $\|P\| \geq 1$ if $P \neq 0$. For subspaces $M, N$ of $X$, we'll say that $P$ is a projection on $M$ along $N$ if $P$ is a projection such that $\operatorname{Im}(P)=M, \operatorname{Ker}(P)=N$.

Given a family of projections $P_{1}, \ldots, P_{N}$ on $M_{1}, \ldots, M_{N}$ in $X$, there is a well known algorithm of finding a projection on $\cap_{j=1}^{N} M_{j}$, which is known as the method of averaged projections. The idea is to define the operator $T=$ $\frac{P_{1}+\ldots+P_{N}}{N}$ and to take a limit $T^{i}$ as $i$ goes to infinity. The reader should note that in general $T^{i}$ need not converge in the operator norm. In [17, the author had established a criterion for the convergence of $T^{i}$ using the idea of an angle between projections. 
Definition 2.10 (Angle between projections). Let $X$ be a Banach space and let $P_{1}, P_{2}$ be projections on $M_{1}, M_{2}$ respectively. Assume that there is a projection $P_{1,2}$ on $M_{1} \cap M_{2}$ such that $P_{1,2} P_{1}=P_{1,2}$ and $P_{1,2} P_{2}=P_{1,2}$ and define

$$
\cos \left(\angle\left(P_{1}, P_{2}\right)\right)=\max \left\{\left\|P_{1}\left(P_{2}-P_{1,2}\right)\right\|,\left\|P_{2}\left(P_{1}-P_{1,2}\right)\right\|\right\} .
$$

Remark 2.11. In the above definition, we are actually defining the "cosine" of the angle. This is a little misleading, because we do not know if $\cos \left(\angle\left(P_{1}, P_{2}\right)\right) \leq$ 1 holds in general (although this inequality holds in all the examples we can compute or bound).

Remark 2.12. We note that in the case where $X$ is a Hilbert space and $P_{1}, P_{2}$ are orthogonal projections on $M_{1}, M_{2}$, the orthogonal projection $P_{1,2}$ on $M_{1} \cap$ $M_{2}$ will always fulfill $P_{1,2} P_{1}=P_{1,2}$ and $P_{1,2} P_{2}=P_{1,2}$. Also, in this case, $\cos \left(\angle\left(P_{1}, P_{2}\right)\right)$ will be equal to the Friedrichs angle between $M_{1}$ and $M_{2}$ defined as

$\cos \left(\angle\left(M_{1}, M_{2}\right)\right)=\sup \left\{|\langle u, v\rangle|:\|u\| \leq 1,\|v\| \leq 1, u \in M_{1} \cap\left(M_{1} \cap M_{2}\right)^{\perp}, v \in M_{2}\right\}$.

Next, we recall the following theorems from [17]:

Theorem 2.13. [17][Theorem 3.12] Let $X$ be a Banach space and let $P_{1}, \ldots, P_{N}$ be projections in $X(N \geq 2)$. Assume that for every $1 \leq j_{1}<j_{2} \leq N$, there is a projection $P_{j_{1}, j_{2}}$ on $\operatorname{Im}\left(P_{j_{1}}\right) \cap \operatorname{Im}\left(P_{j_{2}}\right)$, such that $P_{j_{1}, j_{2}} P_{j_{1}}=P_{j_{1}, j_{2}}, P_{j_{1}, j_{2}} P_{j_{2}}=$ $P_{j_{1}, j_{2}}$.

Denote $T=\frac{P_{1}+\ldots+P_{N}}{N}$ and assume there are constants

$$
\gamma<\frac{1}{8 N-11} \text { and } \beta<1+\frac{1-(8 N-11) \gamma}{N-2+(3 N-4) \gamma}
$$

such that

$$
\max \left\{\left\|P_{1}\right\|, \ldots,\left\|P_{N}\right\|\right\} \leq \beta \text { and } \max \left\{\cos \left(L\left(P_{j_{1}}, P_{j_{2}}\right)\right): 1 \leq j_{1}<j_{2} \leq N\right\} \leq \gamma .
$$

Then for

$$
\begin{gathered}
r=\frac{1+(N-2) \beta}{N}+\left(4-\frac{6}{N}\right) \frac{1+\beta}{1-\gamma} \gamma, \\
C=\frac{(2 N-2) \beta^{2}}{N(1-r)}
\end{gathered}
$$

we have that $r<1$ and there is an operator $T^{\infty}$, such that $\left\|T^{\infty}-T^{i}\right\| \leq C r^{i-1}$. Moreover, $T^{\infty}$ is a projection on $\bigcap_{j=1}^{N} \operatorname{Im}\left(P_{j}\right)$.

To avoid carrying messy constants, we note the following:

Corollary 2.14. In the notations of the above theorem, there are $\gamma_{0}>0$ and $\beta_{0}>1$ such that if

$\max \left\{\left\|P_{1}\right\|, \ldots,\left\|P_{N}\right\|\right\} \leq \beta_{0}$ and $\max \left\{\cos \left(\angle\left(P_{j_{1}}, P_{j_{2}}\right)\right): 1 \leq j_{1}<j_{2} \leq N\right\} \leq \gamma_{0}$,

then $\left\|T^{\infty}-T^{i}\right\| \leq(4 N)\left(\frac{2 N-1}{2 N}\right)^{i-1}$. 
Proof. Note that in Theorem 2.13 above, $r$ tends to $\frac{1+(N-2) \beta}{N}$ as $\gamma$ tends to 0 . Therefore, we can choose $\beta_{0}>1$ and $\gamma_{0}$ small enough such that $r \leq \frac{2 N-1}{2 N}$. Also note that for such $r$, we have that $C=\frac{(2 N-2) \beta_{0}^{2}}{N \frac{1}{2 N}}=(4 N-4) \beta_{0}^{2}$. Therefore, we can choose $\beta_{0}>1$ small enough such that $C \leq 4 N$.

Last, we note that $T^{i}$ converges to a "canonical" projection with respect to $P_{1}, \ldots, P_{N}$ if such projection exists.

Proposition 2.15. Let $X$ be a Banach space and let $P_{1}, \ldots, P_{N}$ be projections in $X(N \geq 2)$. Denote $T=\frac{P_{1}+\ldots+P_{N}}{N}$ and assume that $T^{i}$ converges in the operator norm to $T^{\infty}$ which is a projection on $\bigcap_{j=1}^{N} \operatorname{Im}\left(P_{j}\right)$. If there is a projection $P_{1,2, \ldots, N}$ on $\bigcap_{j=1}^{N} \operatorname{Im}\left(P_{j}\right)$ such that for every $i, P_{1,2, \ldots, N} P_{j}=P_{1,2, \ldots, N}$, then $T^{\infty}=P_{1,2, \ldots, N}$.

Proof. Note that for every $i$, we have that $P_{1, \ldots, N} T^{i}=P_{1, \ldots, N}$ and therefore $T^{\infty}=P_{1, \ldots, N} T^{\infty}=P_{1, \ldots, N}$.

\section{$2.3 \quad$ Type and cotype}

Let $X$ be a Banach space. For $1<p_{1} \leq 2, X$ is said to have (Gaussian) type $p_{1}$, if there is a constant $T_{p_{1}}$, such that for $g_{1}, \ldots, g_{n}$ independent standard Gaussian random variables on a probability space $(\Omega, P)$, we have that for every $x_{1}, \ldots, x_{n} \in X$ the following holds:

$$
\left(\int_{0}^{1}\left\|\sum_{i=1} g_{i}(\omega) x_{i}\right\|^{2} d P\right)^{\frac{1}{2}} \leq T_{p_{1}}\left(\sum_{i=1}^{n}\left\|x_{i}\right\|^{p_{1}}\right)^{\frac{1}{p_{1}}} .
$$

The minimal constant $T_{p_{1}}$ such that this inequality is fulfilled is denoted $T_{p_{1}}(X)$.

For $2 \leq p_{2}<\infty, X$ is said to have (Gaussian) cotype $p_{2}$, if there is a constant $C_{p_{2}}$, such that for $g_{1}, \ldots, g_{n}$ independent standard Gaussian random variables on a probability space $(\Omega, P)$, we have that for every $x_{1}, \ldots, x_{n} \in X$ the following holds:

$$
C_{p_{2}}\left(\sum_{i=1}^{n}\left\|x_{i}\right\|^{p_{2}}\right)^{\frac{1}{p_{2}}} \leq\left(\int_{0}^{1}\left\|\sum_{i=1} g_{i}(\omega) x_{i}\right\|^{2} d P\right)^{\frac{1}{2}} .
$$

The minimal constant $C_{p_{2}}$ such that this inequality is fulfilled is denoted $C_{p_{2}}(X)$.

We recall the following fact mentioned in the introduction regarding $\mathrm{Ba}-$ nach spaces with given type and cotype which is due to Tomczak-Jaegermann 24][Theorem 2 and the corollary after it]: if $X$ is a Banach space of type $p_{1}$, cotype $p_{2}$ and corresponding constants $T_{p_{1}}(X), C_{p_{2}}(X)$ as above, then $d_{k}(X) \leq 4 T_{p_{1}}(X) C_{p_{2}}(X) k^{\frac{1}{p_{1}}-\frac{1}{p_{2}}}$.

Remark 2.16. We remark that the Gaussian type and cotype defined above are equivalent to the usual (Rademacher) type and cotype (see [18] [pages 311-312] and reference therein).

Remark 2.17. In [20], Pisier and Xu showed that for any $p_{2}>2$ one can construct a non superreflexive Banach space $X$ with type 2 and cotype $p_{2}$. 


\subsection{Vector valued $L^{2}$ spaces}

Given a measure space $(\Omega, \mu)$ and Banach space $X$, a function $s: \Omega \rightarrow X$ is called simple if it is of the form:

$$
s(\omega)=\sum_{i=1}^{n} \chi_{E_{i}}(\omega) v_{i}
$$

where $\left\{E_{1}, \ldots, E_{n}\right\}$ is a partition of $\Omega$ where each $E_{i}$ is a measurable set, $\chi_{E_{i}}$ is the indicator function on $E_{i}$ and $v_{i} \in X$.

A function $f: \Omega \rightarrow X$ is called Bochner measurable if it is almost everywhere the limit of simple functions. Denote $L^{2}(\Omega ; X)$ to be the space of Bochner measurable functions such that

$$
\forall f \in L^{2}(\Omega ; X),\|f\|_{L^{2}(\Omega ; X)}=\left(\int_{\Omega}\|f(\omega)\|_{X}^{2} d \mu(\omega)\right)^{\frac{1}{2}}<\infty .
$$

Given an operator $T \in B\left(L^{2}(\Omega, \mu)\right)$, we can define $T \otimes i d_{X} \in B\left(L^{2}(\Omega ; X)\right)$ by defining it first on simple functions.

For our uses, it will be important to bound the norm of an operator of the form $T \otimes i d_{X}$ given that $X$ is derived by one of the deformation procedures given in the introduction.

We will start by bounding the norm of $T \otimes i d_{X}$ given that $X$ has "round" enough finite dimensional subspaces. For this, following [7], we introduce the following notation: for a Banach space $X$ and a constant $k \in \mathbb{N}$ denote

$$
e_{k}(X)=\sup \left\{\left\|T \otimes i d_{X}\right\|_{L^{2}(\Omega ; X)}: T \text { is of rank } k \text { with }\|T\|_{\ell_{2}} \leq 1\right\} .
$$

By a theorem of Pisier (see 7][Theorem 5.2]), this constant is connected to the constant $d_{k}(X)$ defined in the introduction by the inequality $e_{k}(X) \leq$ $2 d_{k}(X)$ (there is also a reverse inequality $d_{k}(X) \leq e_{k}(X)$ which we will not use). Next, we recall the following definition:

Definition 2.18. For a Hilbert space $H$ and a bounded operator $T \in B(H)$ and a constant $r \in[1, \infty]$, the $r$-th Schatten norm is defined as

$$
\begin{gathered}
r<\infty,\|T\|_{S^{r}}=\left(\sum_{i=1}^{\infty}\left(s_{i}(T)\right)^{r}\right)^{\frac{1}{r}}, \\
\|T\|_{S^{\infty}}=s_{1}(T),
\end{gathered}
$$

where $s_{1}(T) \geq s_{2}(T) \geq \ldots$ are the eigenvalues of $\sqrt{T^{*} T}$. An operator $T$ is said to be of Schatten class $r$ if $\|T\|_{S^{r}}<\infty$.

In 22] the following connection was between $e_{k}(X)$ and the norm of $T \otimes i d_{X}$ :

Lemma 2.19. [22][Proposition 3.3] Let $r \in[2, \infty), r>r^{\prime} \geq 2$ be constants and assume there is a constant $C^{\prime}$ such that $e_{k}(X) \leq C^{\prime} k^{\frac{1}{r}}$ for every $k$. Denote

$$
M=\sum_{i=1}^{\infty} 2^{\frac{r^{\prime}}{r^{\prime}-1}\left(\frac{1}{r}-\frac{1}{r^{\prime}}\right) i}
$$

If $(\Omega, \mu)$ is a measure space and $T \in B\left(L^{2}(\Omega, \mu)\right)$ is of Schatten class $r^{\prime}$, then

$$
\left\|T \otimes i d_{X}\right\|_{B\left(L^{2}(\Omega ; X)\right)} \leq M C^{\prime}\|T\|_{S^{r^{\prime}}} .
$$


Remark 2.20. The statement of [22][Proposition 3.3] refers to Banach spaces with specified type and cotype, but is only uses the fact that for these spaces $e_{k}(X)$ can be bounded by some $C^{\prime} k^{\frac{1}{r}}$. Therefore the proof of [22][Proposition 3.3] actually prove the more general case stated above (this was already observed and used in [7]).

Combining the above lemma with the theorem of Pisier stated above gives the following corollary:

Corollary 2.21. Let $r \in[2, \infty), r>r^{\prime}$ be constants and assume there is a constant $C_{1}$ such that $d_{k}(X) \leq C_{1} k^{\frac{1}{r}}$ for every $k$. Then there is a constant $C=$ $C\left(C_{1}, r, r^{\prime}\right)$ such that for every measure space $(\Omega, \mu)$ and every $T \in B\left(L^{2}(\Omega, \mu)\right)$ of Schatten class $r^{\prime}$, we have that

$$
\left\|T \otimes i d_{X}\right\|_{B\left(L^{2}(\Omega ; X)\right)} \leq C\|T\|_{S^{r^{\prime}}}
$$

Second, we will see that if $X$ is given as an interpolation of two spaces $X_{0}, X_{1}$, the norm of $T \otimes i d_{X}$ can be bounded using bounds on the norms of $T \otimes i d_{X_{0}}, T \otimes i d_{X_{1}}:$

Lemma 2.22. 220/Lemma 3.1] Given a compatible pair $\left(X_{0}, X_{1}\right)$, a measure space $(\Omega, \mu)$ and an operator $T \in B\left(L^{2}(\Omega, \mu)\right)$, we have for every $0 \leq \theta \leq 1$ that

$\left\|T \otimes i d_{\left[X_{0}, X_{1}\right]_{\theta}}\right\|_{B\left(L^{2}\left(\Omega ;\left[X_{0}, X_{1}\right]_{\theta}\right)\right)} \leq\left\|T \otimes i d_{X_{0}}\right\|_{B\left(L^{2}\left(\Omega ; X_{0}\right)\right)}^{1-\theta}\left\|T \otimes i d_{X_{1}}\right\|_{B\left(L^{2}\left(\Omega ; X_{1}\right)\right)}^{\theta}$, where $\left[X_{0}, X_{1}\right]_{\theta}$ is the interpolation of $X_{0}$ and $X_{1}$ (see definition above).

Third, if $X$ and $X^{\prime}$ are isomorphic then the norm on $T \otimes i d_{X}$ can be bounded using the norm on $T \otimes i d_{X^{\prime}}$ and the Banach-Mazur distance between $X$ and $X^{\prime}$.

Lemma 2.23. [17][Lemma 2.7] Let $(\Omega, \mu)$ be a measure space and $T$ a bounded operator on $L^{2}(\bar{\Omega}, \mu)$. Given two isomorphic Banach spaces $X, X^{\prime}$, we have that

$$
\left\|T \otimes i d_{X}\right\|_{B\left(L^{2}(\Omega ; X)\right)} \leq d_{B M}\left(X, X^{\prime}\right)\left\|T \otimes i d_{X^{\prime}}\right\|
$$

Last, we need the following fact of regarding passing to the closure under quotients, subspaces, $l_{2}$-sums and ultraproducts:

Lemma 2.24. [22][Lemma 3.1] Let $(\Omega, \mu)$ be a measure space, $C \geq 0$ and $T$ a bounded operator on $L^{2}(\Omega, \mu)$. The class of Banach spaces $X$, for which $\left\|T \otimes i d_{X}\right\| \leq C$ is stable under quotients, subspaces, $l_{2}$-sums and ultraproducts.

Remark 2.25. The fact that the above class is closed under $l_{2}$ sums, did not appear in [22][Lemma 3.1] and it is left as an exercise to the reader.

Combining all the results above yields the following:

Corollary 2.26. Let $T \in B\left(L^{2}(\Omega, \mu)\right)$ be an operator and let $L \geq 1, r^{\prime} \geq 2$ be constants such that $\|T\|_{S^{r^{\prime}}} \leq 1$ and such that for every Banach space $X$ we have that $\left\|T \otimes i d_{X}\right\|_{B\left(L^{2}(\Omega ; X)\right)} \leq L$. Then for every constants $r>r^{\prime}, C_{1} \geq$ $1,1 \geq \theta_{2}>0, C_{3} \geq 1$, there is a constant $C=C\left(C_{1}, r, r^{\prime}\right)$ such that for every $X \in \overline{\mathcal{E}_{3}\left(\mathcal{E}_{2}\left(\mathcal{E}_{1}\left(r, C_{1}\right), \theta_{2}\right), C_{3}\right)}$ the following holds

$$
\left\|T \otimes i d_{X}\right\|_{B\left(L^{2}(\Omega ; X)\right)} \leq C_{3} L\left(C\|T\|_{S^{r^{\prime}}}\right)^{\theta_{2}} .
$$


Proof. By Corollary 2.21 there is a constant $C=C\left(C_{1}, r, r^{\prime}\right)$ such that for every $X \in \mathcal{E}_{1}\left(r, C_{1}\right)$ the following holds:

$$
\left\|T \otimes i d_{X}\right\|_{B\left(L^{2}(\Omega ; X)\right)} \leq C\|T\|_{S^{r^{\prime}}} .
$$

Combining this with Lemma 2.22 and our assumptions on $T$ gives that for every $X \in \mathcal{E}_{2}\left(\mathcal{E}_{1}\left(r, C_{1}\right), \theta_{2}\right)$, we have that

$$
\left\|T \otimes i d_{X}\right\|_{B\left(L^{2}(\Omega ; X)\right)} \leq L\left(C\|T\|_{S^{r^{\prime}}}\right)^{\theta_{2}} .
$$

Applying Lemma 2.23 yields that for every $X \in \mathcal{E}_{3}\left(\mathcal{E}_{2}\left(\mathcal{E}_{1}\left(r, C_{1}\right), \theta_{2}\right), C_{3}\right)$, we have that

$$
\left\|T \otimes i d_{X}\right\|_{B\left(L^{2}(\Omega ; X)\right)} \leq C_{3} L^{1-\theta_{2}}\left(C\|T\|_{S^{r^{\prime}}}\right)^{\theta_{2}} .
$$

Last, Lemma 2.24 states that this inequality does not change when passing to the closure.

\subsection{Group representations in a Banach space}

Let $G$ be a locally compact group and $X$ a Banach space. Let $\pi$ be a representation $\pi: G \rightarrow \mathcal{B}(X)$. Throughout this paper we shall always assume $\pi$ is continuous with respect to the strong operator topology without explicitly mentioning it.

Denote by $C_{c}(G)$ the groups algebra of compactly supported simple functions on $G$ with convolution. For any $f \in C_{c}(G)$ we can define $\pi(f) \in \mathcal{B}(X)$ as

$$
\forall v \in X, \pi(f) \cdot v=\int_{G} f(g) \pi(g) \cdot v d \mu(g),
$$

where the above integral is the Bochner integral with respect to the (left) Haar measure $\mu$ of $G$.

Recall that given $\pi$ one can define the following representations:

1. The complex conjugation of $\pi$, denoted $\bar{\pi}: G \rightarrow \mathcal{B}(\bar{X})$ is defined as

$$
\bar{\pi}(g) \cdot \bar{v}=\overline{\pi(g) \cdot v}, \forall g \in G, \bar{v} \in \bar{X} .
$$

2. The dual representation $\pi^{*}: G \rightarrow \mathcal{B}\left(X^{*}\right)$ is defined as

$$
\left\langle v, \pi^{*}(g) u\right\rangle=\left\langle\pi\left(g^{-1}\right) \cdot v, u\right\rangle, \forall g \in G, v \in X, u \in X^{*} .
$$

Next, we'll restrict ourselves to the case of compact groups. Let $K$ be a compact group with a Haar measure $\mu$ and let $C_{c}(K)=C(K)$ defined as above. Let $X$ be Banach space and let $\pi$ be a representation of $K$ on $X$ that is continuous with respect to the strong operator topology. We shall show that for every $f \in C_{c}(K)$, we can bound the norm of $\pi(f)$ using the norm of $\lambda \otimes i d_{X} \in B\left(L^{2}(K ; X)\right)$ (the definition of $L^{2}(K ; X)$ is given in subsection 2.4 above).

Proposition 2.27. 177][Corollary 2.11] Let $\pi$ be a representation of a compact group $K$ on a Banach space $X$. Then for any real function $f \in C_{c}(G)$ we have that

$$
\|\pi(f)\|_{B(X)} \leq\left(\sup _{g \in K}\|\pi(g)\|\right)^{2}\left\|\left(\lambda \otimes i d_{X}\right)(f)\right\|_{B\left(L^{2}(K ; X)\right)},
$$

where $\lambda$ is the left regular representation of $G$. 


\subsection{Asplund spaces}

Definition 2.28. A Banach space $X$ is said to be an Asplund space if every separable subspace of $X$ has a separable dual.

There are many examples of Asplund spaces - for instance every reflexive space is Asplund. A very nice exposition of Asplund spaces was given by Yost in 26. The reason we are interested in Asplund space is the following theorem of Megrelishvili:

Theorem 2.29. 14][Corollary 6.9] Let $G$ be a topological group and let $\pi$ be a continuous representation of $G$ on a Banach space $X$. If $X$ is an Asplund space, then the dual representation $\pi^{*}$ is also continuous.

\section{Angle between more than 2 projections and space decomposition}

The aim of this section is to show that given several projections on a Banach space, this space can be decomposed with respect to these projections, given that the angle between every two projections is large enough. The main motivation for establishing such a decomposition is applying it to deduce vanishing of cohomology relying on Theorem 2.5. In order to prove this decomposition, we define and study the notion an angle between several projections.

Following our main motivation, we will think about our projections as defined by faces of a simplex:

Definition 3.1. Let $X$ be a Banach space and let $\triangle=\{0, \ldots, n\}$ be a simplex with $n+1$ vertices. For $k=-1,0, \ldots, n$, denote by $\triangle(k)$ the $k$-dimensional faces of $\triangle$, i.e., the subsets of $\triangle$ with cardinality $k+1$.

Let $P_{\sigma}$ be projections defined for every $\sigma \in \triangle(n) \cup \triangle(n-1)$ such that

$$
\forall \sigma \in \triangle(n-1), P_{\sigma} P_{\triangle}=P_{\sigma} .
$$

For every $\tau \subseteq \triangle$ define an operator $T_{\sigma}$ as follows:

$$
T_{\tau}=\left\{\begin{array}{ll}
P_{\triangle} & \tau=\triangle \\
\frac{\sum_{\sigma \in \triangle(n-1), \tau \subseteq \sigma} P_{\sigma}}{|\triangle \backslash \tau|} & \tau \neq \triangle
\end{array} .\right.
$$

Fix $\tau \varsubsetneqq \triangle$. If $T_{\tau}^{i}$ converges to a projection on the space $\cap_{\sigma \in \triangle(n-1), \tau \subseteq \sigma} \operatorname{Im}\left(P_{\sigma}\right)$ as $i \rightarrow \infty$, then we define $P_{\tau}=\lim T_{\tau}^{i}$. In this case we say that $P_{\tau}$ exists.

Remark 3.2. We note that the above setting is general for any $n+1$ projections $P_{0}, \ldots, P_{n}$. Indeed, given any such projections, we can always denote $P_{i}=P_{\triangle \backslash\{i\}}$ and take $P_{\triangle}=I$ (the reason we define the operator $P_{\triangle}$ above is that in the setting we will consider, such an operator appears naturally).

Remark 3.3. By the definition of $P_{\triangle}$, we have for every $\tau \subseteq \triangle$ and every $i$ that

$$
T_{\tau}^{i} P_{\triangle}=T_{\tau}^{i} P_{\triangle}=T_{\tau}^{i}
$$

Therefore for every $\tau \subseteq \triangle$, if $P_{\tau}$ exists, then $P_{\tau} P_{\triangle}=P_{\tau}$. 
Using this notations, we will define the cos of an angle between more than 2 projections:

Definition 3.4. Let $X$ and $P_{\sigma}$ for $\sigma \in \triangle(n-1)$ be defined as in definition 3.1 above. Fix $1 \leq k \leq n$. Denote Sym $(0,1, \ldots, k)$ to be the group of all permutations of $\{0,1, \ldots, k\}$.

For $\sigma_{0}, \ldots, \sigma_{k} \in \triangle(n-1)$ pairwise disjoint, denote $\tau=\bigcap_{i=0}^{k} \sigma_{i}$. If $P_{\tau}$ exists, define $\cos \left(\angle\left(P_{\sigma_{0}}, \ldots, P_{\sigma_{k}}\right)\right)$ as

$$
\cos \left(\angle\left(P_{\sigma_{0}}, \ldots, P_{\sigma_{k}}\right)\right)=\max _{\pi \in \operatorname{Sym}(0, \ldots, k)}\left\|P_{\sigma_{\pi(0)}} P_{\sigma_{\pi(1)}} \ldots P_{\sigma_{\pi(k)}}\left(I-P_{\tau}\right)\right\| .
$$

Theorem 3.5. Let $X, \triangle, P_{\sigma}$ for $\sigma \in \triangle(n-1)$ be defined as above and assume $n>1$. Assume that for every $\eta \in \triangle(n-2)$, the projection $P_{\eta}$ exists and that

$$
\forall \sigma \in \triangle(n-1), \eta \subseteq \sigma \Rightarrow P_{\eta} P_{\sigma}=P_{\eta} .
$$

Also assume that $\max _{\sigma \in \triangle(n-1)}\left\|P_{\sigma}\right\| \leq \beta_{0}$, where $\beta_{0}>1$ is the constant of Corollary 2.14

Then for every $\varepsilon>0$ there is $\gamma>0$ such that if

$$
\max \left\{\cos \left(\angle\left(P_{\sigma}, P_{\sigma^{\prime}}\right)\right): \sigma, \sigma^{\prime} \in \triangle(n-1)\right\} \leq \gamma .
$$

then for every $\tau \subseteq \triangle, P_{\tau}$ is well defined and for every pairwise disjoint $\sigma_{0}, \ldots, \sigma_{k} \in$ $\triangle(n-1)$ the following holds:

$$
\cos \left(\angle\left(P_{\sigma_{0}}, \ldots, P_{\sigma_{k}}\right)\right) \leq \varepsilon .
$$

Proof. Let $\gamma_{0}>0$ and $\beta_{0}$ be the constants of Corollary 2.14 and fix $\varepsilon>0$. Note that $\beta_{0} \leq 2$.

Fix $1 \leq k \leq n$ and $\sigma_{0}, \ldots, \sigma_{k} \in \triangle(n-1)$. Denote $\tau=\cap_{j=0}^{k} \sigma_{j}$.

Assume first that $\gamma \leq \gamma_{0}$, then by Corollary 2.14, we have that $T_{\tau}^{i}$ converges to $P_{\tau}$ and

$$
\left\|P_{\tau}-T_{\tau}^{i}\right\| \leq 4(k+1)\left(\frac{2(k+1)-1}{2(k+1)}\right)^{i-1} \leq 4(n+1)\left(\frac{2(n+1)-1}{2(n+1)}\right)^{i-1} .
$$

Without loss of generality, it is enough to show that there is $\gamma$ such that

$$
\left\|P_{\sigma_{0}} \ldots P_{\sigma_{k}}\left(I-P_{\tau}\right)\right\| \leq \varepsilon .
$$

By (11), we can choose $i_{0}$ large enough such that

$$
\left\|P_{\tau}-T_{\tau}^{i_{0}}\right\| \leq \frac{\varepsilon}{2^{n+2}},
$$

and this $i_{0}$ can be chosen independently of $k$.

Therefore

$$
\begin{array}{r}
\left\|P_{\sigma_{0}} \ldots P_{\sigma_{k}}\left(I-P_{\tau}\right)\right\| \leq\left\|P_{\sigma_{0}} \ldots P_{\sigma_{k}}\left(I-T_{\tau}^{i_{0}}\right)\right\|+\left\|P_{\sigma_{0}} \ldots P_{\sigma_{k}}\left(T_{\tau}^{i_{0}}-P_{\tau}\right)\right\| \leq \\
\left\|P_{\sigma_{0}} \ldots P_{\sigma_{k}}\left(I-T_{\tau}^{i_{0}}\right)\right\|+\left\|P_{\sigma_{0}}\right\| \ldots P_{\sigma_{k}} \| \frac{\varepsilon}{2^{n+2}} \leq \\
\left\|P_{\sigma_{0}} \ldots P_{\sigma_{k}}\left(I-T_{\tau}^{i_{0}}\right)\right\|+\beta_{0}^{k+1} \frac{\varepsilon}{2^{n+2}} \leq \\
\left\|P_{\sigma_{0}} \ldots P_{\sigma_{k}}\left(I-T_{\tau}^{i_{0}}\right)\right\|+\frac{\varepsilon}{2} .
\end{array}
$$


We are left to show that by choosing $\gamma$ small enough, we can ensure that

$$
\left\|P_{\sigma_{0}} \ldots P_{\sigma_{k}}\left(I-T_{\tau}^{i_{0}}\right)\right\| \leq \frac{\varepsilon}{2}
$$

Denote $T_{\tau}^{\prime}=I-T_{\tau}=\frac{\left(I-P_{\sigma_{0}}\right)+\ldots+\left(I-P_{\sigma_{k}}\right)}{k+1}$. Note that

$$
I-T_{\tau}^{i_{0}}=T_{\tau}^{\prime}\left(\left(\begin{array}{c}
i_{0} \\
1
\end{array}\right) I-\left(\begin{array}{c}
i_{0} \\
2
\end{array}\right) T_{\tau}^{\prime}+\ldots+(-1)^{i_{0}-1}\left(\begin{array}{c}
i_{0} \\
i_{0}
\end{array}\right)\left(T_{\tau}^{\prime}\right)^{i_{0}-1}\right) .
$$

Recall that by our assumptions $\left\|T_{\tau}\right\| \leq \beta_{0}$ and therefore that $\left\|T_{\tau}^{\prime}\right\| \leq 1+\beta_{0} \leq 3$. This yields that

$$
\begin{aligned}
& \left\|P_{\sigma_{0}} \ldots P_{\sigma_{k}}\left(I-T_{\tau}^{i_{0}}\right)\right\| \leq \\
& \left\|P_{\sigma_{0}} \ldots P_{\sigma_{k}} T_{\tau}^{\prime}\right\|\left\|\left(\begin{array}{c}
i_{0} \\
1
\end{array}\right) I-\left(\begin{array}{c}
i_{0} \\
2
\end{array}\right) T_{\tau}^{\prime}+\ldots+(-1)^{i_{0}-1}\left(\begin{array}{c}
i_{0} \\
i_{0}
\end{array}\right)\left(T_{\tau}^{\prime}\right)^{i_{0}-1}\right\| \leq \\
& \left\|P_{\sigma_{0}} \ldots P_{\sigma_{k}} T_{\tau}^{\prime}\right\|\left(\|I\|+\left(\begin{array}{c}
i_{0} \\
2
\end{array}\right)\left\|T_{\tau}^{\prime}\right\|+\ldots+\left(\begin{array}{c}
i_{0} \\
i_{0}
\end{array}\right)\left\|T_{\tau}^{\prime}\right\|^{i_{0}-1}\right) \leq \\
& \left\|P_{\sigma_{0}} \ldots P_{\sigma_{k}} T_{\tau}^{\prime}\right\| \frac{1}{3}\left(3+\left(\begin{array}{c}
i_{0} \\
2
\end{array}\right) 3^{2}+\ldots+\left(\begin{array}{c}
i_{0} \\
i_{0}
\end{array}\right) 3^{i_{0}}\right) \leq\left\|P_{\sigma_{0}} \ldots P_{\sigma_{k}} T_{\tau}^{\prime}\right\| \frac{4^{i_{0}}}{3} .
\end{aligned}
$$

Therefore it is enough to show we can choose $\gamma$ small enough such that

$$
\left\|P_{\sigma_{0}} \ldots P_{\sigma_{k}} T_{\tau}^{\prime}\right\| \leq \frac{3}{4^{i_{0}}} \frac{\varepsilon}{2}
$$

(note that $i_{0}$ is independent of $\gamma$ as long as $\gamma \leq \gamma_{0}$ ). We will finish the proof by showing that

$$
\left\|P_{\sigma_{0}} \ldots P_{\sigma_{k}} T_{\tau}^{\prime}\right\| \leq n 2^{n+1} \gamma .
$$

By the definition of $T_{\tau}^{\prime}$, we have that

$$
\left\|P_{\sigma_{0}} \ldots P_{\sigma_{k}} T_{\tau}^{\prime}\right\| \leq\left\|\frac{P_{\sigma_{0}} \ldots P_{\sigma_{k}}\left(I-P_{\sigma_{0}}\right)}{k+1}\right\|+\ldots+\left\|\frac{P_{\sigma_{0}} \ldots P_{\sigma_{k}}\left(I-P_{\sigma_{k}}\right)}{k+1}\right\| .
$$

Therefore, in order to prove inequality (2), it is enough to show that for every $j, k$ such that $k \geq j \geq 0$, we have that

$$
\left\|P_{\sigma_{0}} \ldots P_{\sigma_{k}}\left(I-P_{\sigma_{j}}\right)\right\| \leq(k-j) 2^{k+1} \gamma .
$$

We will show this by induction on $k-j$. If $k-j=0$, i.e., if $k=j$ then

$$
P_{\sigma_{0}} \ldots P_{\sigma_{k}}\left(I-P_{\sigma_{k}}\right)=0
$$

and we are done. Assume that $k>j$ and that the inequality holds for $k-1, j$, i.e., assume that

$$
\left\|P_{\sigma_{0}} \ldots P_{\sigma_{k-1}}\left(I-P_{\sigma_{j}}\right)\right\| \leq(k-1-j) 2^{k} \gamma
$$

Then for $k$ and $j$ we have that

$$
\begin{aligned}
P_{\sigma_{0}} \ldots P_{\sigma_{k}}\left(I-P_{\sigma_{j}}\right) & =P_{\sigma_{0}} \ldots P_{\sigma_{k-1}}\left(P_{\sigma_{k}}-P_{\sigma_{k}} P_{\sigma_{j}}\right) \\
& =P_{\sigma_{0}} \ldots P_{\sigma_{k-1}}\left(P_{\sigma_{k}}-P_{\sigma_{j}} P_{\sigma_{k}}\right)+P_{\sigma_{0}} \ldots P_{\sigma_{k-1}}\left(P_{\sigma_{j}} P_{\sigma_{k}}-P_{\sigma_{k}} P_{\sigma_{j}}\right) \\
& =P_{\sigma_{0}} \ldots P_{\sigma_{k-1}}\left(I-P_{\sigma_{j}}\right) P_{\sigma_{k}}+P_{\sigma_{0}} \ldots P_{\sigma_{k-1}}\left(P_{\sigma_{j}} P_{\sigma_{k}}-P_{\sigma_{k}} P_{\sigma_{j}}\right) .
\end{aligned}
$$


Therefore

$$
\begin{aligned}
& \left\|P_{\sigma_{0}} \ldots P_{\sigma_{k}}\left(I-P_{\sigma_{j}}\right)\right\| \leq \\
& \quad\left\|P_{\sigma_{0}} \ldots P_{\sigma_{k-1}}\left(I-P_{\sigma_{j}}\right) P_{\sigma_{k}}\right\|+\left\|P_{\sigma_{0}} \ldots P_{\sigma_{k-1}}\left(P_{\sigma_{j}} P_{\sigma_{k}}-P_{\sigma_{k}} P_{\sigma_{j}}\right)\right\| .
\end{aligned}
$$

Note that

$$
\left\|P_{\sigma_{j}} P_{\sigma_{k}}-P_{\sigma_{k}} P_{\sigma_{j}}\right\| \leq\left\|P_{\sigma_{j}} P_{\sigma_{k}}-P_{\sigma_{k} \cap \sigma_{j}}\right\|+\left\|P_{\sigma_{k}} P_{\sigma_{j}}-P_{\sigma_{k} \cap \sigma_{j}}\right\| \leq 2 \gamma,
$$

and therefore

$$
\left\|P_{\sigma_{0}} \ldots P_{\sigma_{k-1}}\left(P_{\sigma_{j}} P_{\sigma_{k}}-P_{\sigma_{k}} P_{\sigma_{j}}\right)\right\| \leq\left\|P_{\sigma_{0}} \ldots P_{\sigma_{k-1}}\right\| 2 \gamma \leq 2^{k+1} \gamma .
$$

Also, note that by the induction assumption

$$
\left\|P_{\sigma_{0}} \ldots P_{\sigma_{k-1}}\left(I-P_{\sigma_{j}}\right) P_{\sigma_{k}}\right\| \leq(k-1-j) 2^{k} \gamma\left\|P_{\sigma_{k}}\right\| \leq(k-1-j) 2^{k+1} \gamma .
$$

Combining the two inequalities above yields

$$
\left\|P_{\sigma_{0}} \ldots P_{\sigma_{k}}\left(I-P_{\sigma_{j}}\right)\right\| \leq(k-j) 2^{k+1} \gamma
$$

as needed.

Definition 3.6 (Consistency). Let $X, \triangle, P_{\sigma}$ for $\sigma \in \triangle(n-1)$ defined as above. We shall say that the projections $P_{\sigma}$ for $\sigma \in \triangle(n-1)$ are consistent, given that for every $\tau \subseteq \eta \varsubsetneqq \triangle$, if $P_{\tau}$ and $P_{\eta}$ exist then $P_{\tau} P_{\eta}=P_{\tau}$.

Remark 3.7. If the projections $P_{\sigma}$ for $\sigma \in \triangle(n-1)$ are consistent and $P_{\tau}$ exists for every $\tau \subseteq \triangle$, then for every $\tau, \tau^{\prime} \subseteq \triangle$, we can define $\cos \left(\angle\left(P_{\tau}, P_{\tau^{\prime}}\right)\right)$ as in the background section, i.e.,

$$
\cos \left(\angle\left(P_{\tau}, P_{\tau^{\prime}}\right)\right)=\max \left\{\left\|P_{\tau} P_{\tau^{\prime}}-P_{\tau \cap \tau^{\prime}}\right\|,\left\|P_{\tau^{\prime}} P_{\tau}-P_{\tau \cap \tau^{\prime}}\right\|\right\} .
$$

Proposition 3.8. Let $X, \triangle, P_{\sigma}$ for $\sigma \in \triangle(n-1)$ defined as above. Assume that for every $\tau \in \triangle, P_{\tau}$ exists. Then the projections $P_{\sigma}$ for $\sigma \in \triangle(n-1)$ are consistent if and only if for

$$
\forall \tau \varsubsetneqq \triangle, \forall \sigma \in \triangle(n-1), \tau \subseteq \sigma \Rightarrow P_{\tau} P_{\sigma}=P_{\tau} .
$$

Proof. One direction is trivial - assume that the projections $P_{\sigma}$ for $\sigma \in \triangle(n-1)$ are consistent, then for every $\tau \subseteq \eta \varsubsetneqq \triangle$, we have that $P_{\tau} P_{\eta}=P_{\tau}$ and in particular this holds for every $\eta \in \triangle(n-1)$.

In the other direction, fix some $\tau \subseteq \eta \varsubsetneqq \triangle$. By our assumptions, we have for every $\sigma \in \triangle(n-1), \tau \subseteq \sigma$ that $P_{\tau} P_{\sigma}=P_{\tau}$. Therefore, by the definition of $T_{\eta}$

$$
\forall i, P_{\tau}\left(T_{\eta}\right)^{i}=P_{\tau}
$$

which in turn implies that $P_{\tau} P_{\eta}=P_{\tau}$ as needed.

Proposition 3.9. Let $X, \triangle, P_{\sigma}$ for $\sigma \in \triangle(n-1)$ defined as above. Assume that for every $\tau \subseteq \triangle, P_{\tau}$ exists. If for every $\tau \varsubsetneqq \triangle$ there is a projection $P_{\tau}^{\prime}$ on $\cap_{\sigma \in \triangle(n-1), \tau \subseteq \sigma} \operatorname{Im}\left(P_{\sigma}\right)$ such that

$$
\forall \sigma \in \triangle(n-1), \tau \subseteq \sigma \Rightarrow P_{\tau}^{\prime} P_{\sigma}=P_{\tau}^{\prime},
$$

then the projections $P_{\sigma}$ for $\sigma \in \triangle(n-1)$ are consistent and for every $\tau \varsubsetneqq \triangle$, $P_{\tau}=P_{\tau}^{\prime}$. 
Proof. By Proposition 2.15, we have that $T_{\tau}^{i}$ converges to $P_{\tau}^{\prime}$ for every $\tau \varsubsetneqq \triangle$ and the consistency follows from Proposition 3.8 .

The main tool that we will use to decompose the space $X$ is the following theorem stating that bounding the angle between each $P_{\sigma}, P_{\sigma^{\prime}}$ where $\sigma, \sigma^{\prime} \in$ $\triangle(n-1)$ gives a bound on the angle between $P_{\tau}, P_{\tau^{\prime}}$ where $\tau, \tau^{\prime}$ are any faces of $\triangle$.

Theorem 3.10. Let $X, \triangle, P_{\triangle}$ and $P_{\sigma}$ for $\sigma \in \triangle(n-1)$ defined as above. Assume the following:

1. The projections $P_{\sigma}$ for $\sigma \in \triangle(n-1)$ are consistent.

2. For any $\eta \in \triangle(n-2)$, the projections $P_{\eta}$ exist.

3. $\max _{\sigma \in \triangle(n-1) \cup \triangle(n)}\left\|P_{\sigma}\right\| \leq \beta_{0}$, where $\beta_{0}>1$ is the constant of Corollary 2.14

Then for every $\varepsilon>0$ there is $\gamma>0$ such that if

$$
\max \left\{\cos \left(\angle\left(P_{\sigma}, P_{\sigma^{\prime}}\right)\right): \sigma, \sigma^{\prime} \in \triangle(n-1)\right\} \leq \gamma .
$$

then the following holds:

1. For every $\tau \subseteq \triangle, P_{\tau}$ exists and $\left\|P_{\tau}\right\| \leq 4(n+1)+2$.

2. For every $\tau, \tau^{\prime} \subseteq \triangle$ and every $\eta \subseteq \triangle$ such that $\tau \cap \tau^{\prime} \subseteq \eta$ we have that

$$
\left\|P_{\tau} P_{\tau^{\prime}}\left(I-P_{\eta}\right)\right\| \leq \varepsilon \text {. }
$$

In particular, $\cos \left(\angle\left(P_{\tau}, P_{\tau^{\prime}}\right)\right) \leq \varepsilon$.

Remark 3.11. Variations of the above theorem were proven in the setting of Hilbert spaces in [8], [9] and [13]. However, all these proofs use the fact that in a Hilbert space the following equality holds for any two subspaces $U, V: \angle(V, U)=$ $\angle\left(V^{\perp}, U^{\perp}\right)$, where the angle here is the Friedrichs angle. In our setting, we do not know if such equality holds, namely if $\cos \left(\angle\left(P_{\tau}, P_{\tau^{\prime}}\right)\right)=\cos \left(\angle\left(I-P_{\tau}, I-\right.\right.$ $\left.P_{\tau^{\prime}}\right)$ ) (we don't even know if $\cos \left(\angle\left(I-P_{\tau}, I-P_{\tau^{\prime}}\right)\right)$ is well defined). This limitation required us to give a more direct proof using the idea of angle between several projections.

Proof. Let $\gamma_{0}>0$ and $\beta_{0}$ be the constants of Corollary 2.14 and let $\varepsilon^{\prime}>0$ be a constant to be determined later. By Theorem 3.5, there is a constant $\gamma_{1}>0$ such that if

$$
\max \left\{\cos \left(\angle\left(P_{\sigma}, P_{\sigma^{\prime}}\right)\right): \sigma, \sigma^{\prime} \in \triangle(n-1)\right\} \leq \gamma,
$$

then for any $k=1, \ldots, n$ and for any $\eta \in \triangle(n-1-k)$, we have that

$$
\cos \left(\angle\left(P_{\sigma_{0}}, \ldots, P_{\sigma_{k}}\right)\right) \leq \varepsilon^{\prime},
$$

where $\sigma_{0}, \ldots, \sigma_{k} \in \triangle(n-1)$ are all the $n-1$ faces of $\triangle$ that contain $\eta$. Choose $\gamma=\min \left\{\gamma_{0}, \gamma_{1}\right\}$.

If $\tau \in \triangle(n-1) \cup \triangle(n)$, then $P_{\tau}$ exists and $\left\|P_{\tau}\right\| \leq \beta_{0}<2 \leq 4(n+1)+2$. Assume next $|\tau|<n$, then by Corollary 2.14 we have that $P_{\sigma}$ is exists and

$$
\left\|P_{\tau}\right\| \leq 4(n+1)+\left\|T_{\tau}\right\| \leq 4(n+1)+\beta_{0} \leq 4(n+1)+2 .
$$


This concludes the proof of the first assertion of the theorem.

Let $\tau, \tau^{\prime} \subseteq \triangle$ and $\eta \subseteq \triangle$ such that $\tau \cap \tau^{\prime} \subseteq \eta$. First, we note that by the consistency assumption $P_{\tau \cap \tau^{\prime}}\left(I-P_{\eta}\right)=0$ and therefore

$$
\begin{aligned}
& \left\|P_{\tau} P_{\tau^{\prime}}\left(I-P_{\eta}\right)\right\|=\left\|P_{\tau} P_{\tau^{\prime}}\left(I-P_{\tau \cap \tau^{\prime}}\right)\left(I-P_{\eta}\right)\right\| \leq \\
& \quad \cos \left(L\left(P_{\tau}, P_{\tau^{\prime}}\right)\right)\left\|I-P_{\eta}\right\| \leq(4(n+1)+3) \cos \left(L\left(P_{\tau}, P_{\tau^{\prime}}\right)\right) .
\end{aligned}
$$

Therefore, it is enough to show that for $\gamma$ small enough

$$
\left\|P_{\tau} P_{\tau^{\prime}}\left(I-P_{\tau \cap \tau^{\prime}}\right)\right\| \leq \frac{\varepsilon}{(4(n+1)+3)},
$$

for any $\tau, \tau^{\prime} \subseteq \triangle$.

Note that if $\tau=\tau^{\prime}$ or $\tau=\triangle$ or $\tau^{\prime}=\triangle$, then $\cos \left(\angle\left(P_{\tau}, P_{\tau^{\prime}}\right)\right)=0$ and there is nothing to prove. Therefore, we can assume that $\tau \cap \tau^{\prime} \in \triangle(n-1-k)$ for $1 \leq k \leq n$. Let $\sigma_{0}, \ldots, \sigma_{k} \in \triangle(n-1)$ be all the pairwise disjoint simplices that contain $\tau \cap \tau^{\prime}$. Without loss of generality we can assume that

$$
\tau \subseteq \sigma_{0}, \ldots, \tau \subseteq \sigma_{j} \text { and } \tau^{\prime} \subseteq \sigma_{j+1}, \ldots, \tau^{\prime} \subseteq \sigma_{k} .
$$

We note that by the consistency assumption

$$
P_{\tau}=P_{\tau} P_{\sigma_{0}} \ldots P_{\sigma_{j}},
$$

and

$$
P_{\tau^{\prime}}=P_{\sigma_{j+1}} \ldots P_{\sigma_{k}} P_{\tau^{\prime}}
$$

Therefore

$$
\begin{aligned}
& \left\|P_{\tau} P_{\tau^{\prime}}\left(I-P_{\tau \cap \tau^{\prime}}\right)\right\|= \\
& \left\|P_{\tau} P_{\sigma_{0}} \ldots P_{\sigma_{k}} P_{\tau^{\prime}}\left(I-P_{\tau \cap \tau^{\prime}}\right)\right\|= \\
& \left\|P_{\tau} P_{\sigma_{0}} \ldots P_{\sigma_{k}}\left(I-P_{\tau \cap \tau^{\prime}}\right) P_{\tau^{\prime}}\right\| \leq \\
& \left\|P_{\tau}\right\|\left\|P_{\tau^{\prime}}\right\| \cos \left(\angle\left(P_{\sigma_{0}}, \ldots, P_{\sigma_{k}}\right)\right) \leq(4(n+1)+2)^{2} \varepsilon^{\prime} .
\end{aligned}
$$

We conclude by choosing $\varepsilon^{\prime}=\frac{\varepsilon}{(4(n+1)+2)^{2}(4(n+1)+3)}$.

Remark 3.12. Theorem 3.10 can be proven without the assumption that the projections $P_{\sigma}$ with $\sigma \in \triangle(n-1)$ are consistent. However, we could not prove Theorem 3.13 below without this assumption (see remark after the proof of Theorem 3.13). Our motivation for proving Theorem 3.10 was deducing Theorem 3.13 and therefore we assumed consistency in the proof (this assumption simplifies the proof considerably). For completeness, we added a proof of Theorem 3.10 in the appendix that does not rely on the consistency assumption.

Assuming that $P_{\eta}$ exists for each $\eta \subseteq \triangle$, we denote $X_{\eta}=\operatorname{Im}\left(P_{\eta}\right)$ and

$$
X^{\eta}=\left\{\begin{array}{ll}
X_{\emptyset} & \eta=\emptyset \\
X_{\eta} \cap \bigcap_{\tau \subsetneq \eta} \operatorname{Ker}\left(P_{\tau}\right) & \eta \neq \emptyset
\end{array} .\right.
$$

The next theorem states that under suitable bounds on the angles between the $P_{\sigma}$ 's for $\sigma \in \triangle(n-1)$ and the norms of the $P_{\sigma}$ 's for $\sigma \in \triangle(n-1) \cup \triangle(n)$, we have that

$$
X_{\eta}=\bigoplus_{\tau \subseteq \eta} X^{\tau} .
$$


Theorem 3.13. Let $X, \triangle, P_{\triangle}$ and $P_{\sigma}$ for $\sigma \in \triangle(n-1)$ defined as above. Assume the following:

1. The projections $P_{\sigma}$ for $\sigma \in \triangle(n-1)$ are consistent.

2. For every $\tau \in \triangle(n-2)$, the projection $P_{\tau}$ exists.

3. $\max _{\sigma \in \triangle(n-1) \cup \triangle(n)}\left\|P_{\sigma}\right\| \leq \beta_{0}$, where $\beta_{0}>1$ is the constant of Corollary 2.14

Then there is $\gamma>0$ such that if

$$
\max \left\{\cos \left(\angle\left(P_{\sigma}, P_{\sigma^{\prime}}\right)\right): \sigma, \sigma^{\prime} \in \triangle(n-1)\right\} \leq \gamma,
$$

then for every $\eta \subseteq \triangle, P_{\eta}$ exists and

$$
X_{\eta}=\bigoplus_{\tau \subseteq \eta} X^{\tau} .
$$

The proof of this theorem is based on a theorem similar to our Theorem 3.10 that appears in 8 [section 11] and the proof given there applies almost verbatim is our setting. We will repeat the proof below for completeness, but we claim no originality here.

Lemma 3.14. Let $X, \triangle, P_{\triangle}$ and $P_{\sigma}$ for $\sigma \in \triangle(n-1)$ defined as above. Assume that the projections $P_{\sigma}$ for $\sigma \in \triangle(n-1)$ are consistent and that for every $\tau \subseteq \triangle, P_{\tau}$ exists.

Fix $0 \leq i \leq n+1$ and assume that for every $\tau \subseteq \triangle$ with $|\tau|<i$ there is a projection $R_{\tau}: X \rightarrow X$ on $X^{\tau}$ such that $R_{\tau}=R_{\tau} P_{\tau}$. Then for every $\eta \subseteq \triangle$ with $|\eta|=i$ the following holds for every $v \in X_{\eta}$ :

$$
v \in X^{\eta} \Leftrightarrow \forall \tau \varsubsetneqq \eta, R_{\tau} v=0 .
$$

Proof. Assume first that $v \in X^{\eta}$, then by definition for every $\tau \varsubsetneqq \eta, v \in$ $\operatorname{Ker}\left(P_{\tau}\right)$. By assumptions of the lemma $R_{\tau} P_{\tau}=R_{\tau}$ and therefore $R_{\tau} v=$ $R_{\tau} P_{\tau} v=0$.

In the other direction we will use induction on $|\eta|$. For $|\eta|=0, X^{\emptyset}=X_{\emptyset}$ and therefore the assertion of the lemma holds. Fix $0<i<n+1$ and assume the lemma is true for every $\tau \subseteq \triangle$ with $|\tau|<i$. Fix $\eta \subseteq \triangle$ with $|\eta|=i$ and fix $v \in X_{\eta}$ such that for every $\tau \varsubsetneqq \eta, R_{\tau} v=0$. Let $\tau \varsubsetneqq \eta$ arbitrary. By the assumptions of the lemma for every $\tau^{\prime} \varsubsetneqq \tau$ the following holds:

$$
R_{\tau^{\prime}} P_{\tau} v=R_{\tau^{\prime}} P_{\tau^{\prime}} P_{\tau} v
$$

By the consistency assumption (and remark 3.3), $P_{\tau^{\prime}} P_{\tau}=P_{\tau^{\prime}}$ and therefore

$$
R_{\tau^{\prime}} P_{\tau} v=R_{\tau^{\prime}} P_{\tau^{\prime}} v=R_{\tau^{\prime}} v=0 .
$$

By the induction assumption, we conclude that $P_{\tau} v \in X^{\tau}$. We also assumed that $R_{\tau} P_{\tau} v=R_{\tau} v=0$, therefore this yields that $P_{\tau} v=0$. We showed that for every $\tau \varsubsetneqq \eta, v \in \operatorname{Ker}\left(P_{\tau}\right)$ which implies that $v \in X^{\eta}$.

We will use the above lemma to prove Theorem 3.13 . 
Proof. Let $\varepsilon>0$ to be determined later and let $\gamma>0$ be the constant corresponding to $\varepsilon>0$ given by Theorem 3.10 .

We shall prove that if $\varepsilon>0$ is small enough, then for each $0 \leq i \leq(n+1)$, there is a constant $C_{i}$ such that the following holds:

1. For each $\eta \subseteq \triangle$ with $|\eta| \leq i$, there is a projection $R_{\eta}: X \rightarrow X$ on $X^{\eta}$ such that $R_{\eta} P_{\eta}=R_{\eta}$ and $\left\|R_{\eta}\right\| \leq C_{i}$.

2. For every $0 \leq j \leq i, C_{i} \geq C_{j}$.

3. For every $\eta, \eta^{\prime} \subseteq \triangle$ such that $\eta \neq \eta^{\prime}$ and $|\eta|,\left|\eta^{\prime}\right| \leq i$, we have that $\left\|R_{\eta} R_{\eta^{\prime}}\right\| \leq\left(C_{i}\right)^{2} \varepsilon$.

4. For each $\eta \subseteq \triangle$ with $|\eta|=i, X_{\eta}=\bigoplus_{\tau \subseteq \eta} X^{\tau}$.

The cases $i=0, i=1$ are straightforward:

For $i=0$, we have that if $|\eta|=0$, then $\eta=\emptyset$. Take $R_{\emptyset}=P_{\emptyset}$ and $C_{0}=$ $4(n+1)+2$. We will check that for this choice conditions 1.-4. hold:

1. Note that

$$
R_{\emptyset} P_{\emptyset}=P_{\emptyset} P_{\emptyset}=P_{\emptyset}=R_{\emptyset} .
$$

Also by Theorem $3.10,\left\|R_{\emptyset}\right\| \leq C_{0}$.

2. Holds vacuously.

3. Holds vacuously.

4. $X_{\emptyset}=X^{\emptyset}$.

Also, by definition $X_{\emptyset}=X^{\emptyset}$.

For $i=1$, for $\eta \subseteq \triangle$ with $|\eta|=1$, take $R_{\eta}=P_{\eta}-P_{\emptyset}$ and $C_{1}=2(4(n+1)+2)$. We will check that for this choice conditions 1.-4. hold:

1. Note that

$$
R_{\eta}=P_{\eta}-P_{\emptyset}=\left(I-P_{\emptyset}\right) P_{\eta}=\left(I-P_{\emptyset}\right) P_{\eta} P_{\eta}=R_{\eta} P_{\eta} .
$$

Also, by Theorem 3.10

$$
\left\|R_{\eta}\right\| \leq\left\|P_{\eta}\right\|+\left\|P_{\emptyset}\right\| \leq C_{1} .
$$

2. $C_{1}=2 C_{0} \geq C_{0}$.

3. Let $\eta, \eta^{\prime} \subseteq \triangle$ such that $|\eta|,\left|\eta^{\prime}\right| \leq 1$ and $\eta \neq \eta^{\prime}$. If $\eta=\emptyset$ or $\eta^{\prime}=\emptyset$, then $R_{\eta} R_{\eta^{\prime}}=0$. If $|\eta|=\left|\eta^{\prime}\right|=1$, then $\eta \cap \eta^{\prime}=\emptyset$ and

$$
\left\|R_{\eta} R_{\eta^{\prime}}\right\|=\left\|\left(P_{\eta}-P_{\emptyset}\right)\left(P_{\eta^{\prime}}-P_{\emptyset}\right)\right\|=\left\|P_{\eta} P_{\eta^{\prime}}-P_{\eta \cap \eta^{\prime}}\right\| \leq \varepsilon \leq C_{1}^{2} \varepsilon,
$$

as needed.

4. For every $\eta \subseteq \triangle$, such that $|\eta|=1, P_{\eta}-P_{\emptyset}$ is a projection on $X^{\eta}$ and therefore $X_{\eta}=X^{\eta} \oplus X^{\emptyset}$. 
We proceed by induction. Let $i>1$ and assume that (1),(2),(3), (4) above hold for every $j<i$.

Step 1 (proof of conditions 1.,2.): Let $\eta \subseteq \triangle$ with $|\eta|=i$. We will show that $X_{\eta}$ is a sum of $X^{\tau}$ with $\tau \subseteq \eta$ and in doing so, we will find a projection operator $R_{\eta}: X \rightarrow X$ such that $\operatorname{Im}\left(R_{\eta}\right)=X^{\eta}$ and $R_{\eta} P_{\eta}=R_{\eta}$.

Let $d=2^{i}-2$ and consider the $(d+1)$-valent tree such that each edge is labelled by some $\tau \varsubsetneqq \eta$ and no two edges with the same label meet at a vertex. Fix a vertex $x_{0}$ to be the root of this tree. Then for every vertex $x_{j}$ with distance $j>0$ from $x_{0}$ there is a path labelled $\tau_{1}, \ldots, \tau_{j}$ from $x_{0}$ to $x_{j}$. For such $x_{j}$, define and operator $R\left(x_{j}\right)=(-1)^{j} R_{\tau_{j}} \ldots R_{\tau_{1}}$ and define $R\left(x_{0}\right)=I$. Denote the vertices of the tree by $V$ and define

$$
L_{\eta}=\sum_{x \in V} R(x) .
$$

Let $x_{j}$ be a vertex with distance $j>0$ from $x_{0}$. By the induction assumption (3) we have that $\left\|R\left(x_{j}\right)\right\| \leq\left(C_{i-1}^{2} \varepsilon\right)^{j-1} C_{i-1}$. Therefore if we choose $\varepsilon \leq \frac{1}{2 d C_{i-1}^{2}}$, then for every $v \in X_{\eta}, \sum_{x} R(x) v$ is absolutely convergent:

$$
\sum_{x}\|R(x) v\|=\left(1+(d+1) C_{i-1} \sum_{j=1}^{\infty}\left(d C_{i-1}^{2} \varepsilon\right)^{j-1}\right)\|v\| \leq\left(1+2(d+1) C_{i-1}\right)\|v\| .
$$

Therefore $L_{\eta}$ is well defined if $\varepsilon$ is sufficiently small. For every $\tau \varsubsetneqq \eta$, denote

$$
\begin{gathered}
B_{\tau}=\left\{x \in V \backslash\left\{x_{0}\right\} \text { such that the path from } x_{0} \text { to } x \text { begins with } \tau\right\}, \\
E_{\tau}=\left\{x \in V \backslash\left\{x_{0}\right\} \text { such that the path from } x_{0} \text { to } x \text { ends with } \tau\right\} .
\end{gathered}
$$

Then for a every $\tau \varsubsetneqq \eta$, we have that

$$
\begin{aligned}
L_{\eta} & =\sum_{x \in E_{\tau}} R(x)+\sum_{x \in V \backslash E_{\tau}} R(x) \\
& =-R_{\tau}\left(\sum_{x \in V \backslash E_{\tau}} R(x)\right)+\sum_{x \in V \backslash E_{\tau}} R(x) .
\end{aligned}
$$

Therefore, for every $\tau \varsubsetneqq \eta, R_{\tau} L_{\eta}=0$ and therefore by Lemma 3.14 above, for every $v \in X_{\eta}, L_{\eta} v \in X^{\eta}$. This shows that $\operatorname{Im}\left(L_{\eta}\right) \subseteq X^{\eta}$. To see that $\operatorname{Im}\left(L_{\eta}\right)=X^{\eta}$, notice that for every $v \in X^{\eta}$ and for every $\tau \varsubsetneqq \eta, R_{\tau} v=0$ and therefore by the definition of $L_{\eta}, L_{\eta} v=v$.

We will take $L_{\eta} P_{\eta}$ as our candidate for $R_{\eta}$ and take $C_{i}=(4(n+1)+2)(1+$ $\left.2(d+1) C_{i-1}\right)$ as a bound on $\left\|R_{\eta}\right\|$ (we showed above that $\left\|L_{\eta}\right\| \leq 1+2(d+1) C_{i-1}$ ). Notice that $C_{i}$ was chosen such that $C_{i} \geq C_{i-1}$ as needed. It is clear that taking $R_{\eta}=L_{\eta} P_{\eta}$ implies that $R_{\eta} P_{\eta}=R_{\eta}$.

To show that $R_{\eta}$ is indeed a projection, notice first that for every $\tau \varsubsetneqq \eta$, we have that

$$
\begin{aligned}
L_{\eta} & =\sum_{x \in B_{\tau}} R(x)+\sum_{x \in V \backslash B_{\tau}} R(x) \\
& =-\left(\sum_{x \in V \backslash B_{\tau}} R(x)\right) R_{\tau}+\sum_{x \in V \backslash B_{\tau}} R(x) .
\end{aligned}
$$


Therefore, for every $\tau \varsubsetneqq \eta, L_{\eta} R_{\tau}=0$. Second, notice that

$$
\begin{aligned}
L_{\eta} & =I+\sum_{\tau \varsubsetneqq \eta} \sum_{x \in E_{\tau}} R(x) \\
& =I-\sum_{\tau \varsubsetneqq \eta} R_{\tau} \sum_{x \in V \backslash E_{\tau}} R(x) .
\end{aligned}
$$

Therefore

$$
L_{\eta}^{2}=L_{\eta}\left(I-\sum_{\tau \varsubsetneqq \eta} R_{\tau} \sum_{x \in V \backslash E_{\tau}} R(x)\right)=L_{\eta}-\sum_{\tau \varsubsetneqq \eta} L_{\eta} R_{\tau} \sum_{x \in V \backslash E_{\tau}} R(x)=L_{\eta} .
$$

This yields that $R_{\eta}^{2}=R_{\eta}$.

The same computation also shows that $X_{\eta}$ is a linear sum of $X^{\tau}$ with $\tau \subseteq \eta$. First, for every $v \in X_{\eta}$ we showed that $L_{\eta} v \in X^{\eta}$. Second, if we denote for every $\tau \varsubsetneqq \eta$,

$$
v^{\tau}=R_{\tau} \sum_{x \in V \backslash E_{\tau}} R(x) v,
$$

then $v^{\tau} \in X^{\tau}$. Last, we showed above that

$$
L_{\eta}=I-\sum_{\tau \varsubsetneqq \eta} R_{\tau} \sum_{x \in V \backslash E_{\tau}} R(x),
$$

and this yields that for every $v \in X_{\eta}$,

$$
v=\sum_{\tau \subsetneq \eta} v^{\tau}+L_{\eta} v
$$

as needed.

Step 2 (proof of condition 3.): We will show that for every $\eta, \eta^{\prime} \subseteq \triangle$, with $|\eta|,\left|\eta^{\prime}\right| \leq i$ and $\eta \neq \eta^{\prime}$, we have that $\left\|R_{\eta} R_{\eta^{\prime}}\right\| \leq\left(C_{i}\right)^{2} \varepsilon$. We'll split the proof of this fact into several cases.

In the case that $\eta \cap \eta^{\prime} \varsubsetneqq \eta^{\prime}$, notice that $\operatorname{Im}\left(P_{\eta^{\prime}}\right) \cap \operatorname{Ker}\left(P_{\eta \cap \eta^{\prime}}\right) \subseteq \operatorname{Im}\left(R_{\eta^{\prime}}\right)$ and therefore

$$
R_{\eta} R_{\eta^{\prime}}=R_{\eta} P_{\eta} P_{\eta^{\prime}}\left(I-P_{\eta \cap \eta^{\prime}}\right) R_{\eta^{\prime}}
$$

This yields that

$$
\left\|R_{\eta} R_{\eta^{\prime}}\right\| \leq\left\|R_{\eta}\right\|\left\|R_{\eta^{\prime}}\right\| \cos \left(\angle\left(P_{\eta}, P_{\eta^{\prime}}\right)\right) \leq C_{|\eta|} C_{\left|\eta^{\prime}\right|} \varepsilon \leq\left(C_{i}\right)^{2} \varepsilon
$$

as needed.

In the case that $\eta \subseteq \eta^{\prime}$, we have that $\operatorname{Im}\left(R_{\eta^{\prime}}\right) \subseteq \operatorname{Ker}\left(P_{\eta}\right)$ and therefore

$$
R_{\eta} R_{\eta^{\prime}}=R_{\eta} P_{\eta} R_{\eta^{\prime}}=0 .
$$

In the case that $\eta^{\prime} \subseteq \eta$ and $|\eta| \leq i-1,\left|\eta^{\prime}\right| \leq i-1$, the inequality follows from the induction assumption.

We are left with the case in which $|\eta|=i$ and $\eta^{\prime} \subseteq \eta$. In this case, by step 1 above, $L_{\eta} R_{\eta^{\prime}}=0$ and therefore $R_{\eta} R_{\eta^{\prime}}=0$ and we are done.

Step 3 (proof of condition 4.): We will finish by showing that given that $\varepsilon>0$ is small enough,

$$
X_{\eta}=\bigoplus_{\tau \subseteq \eta} X^{\tau}
$$


We already showed in step 1 , that $X_{\eta}$ is a linear sum of $X^{\tau}$ such that $\tau \subseteq \eta$. Assume there are $v^{\tau} \in X^{\tau}$ such that

$$
\sum_{\tau \subseteq \eta} v^{\tau}=0 .
$$

Let $\tau^{\prime}$ be such that for every $\tau \subseteq \sigma,\left\|v^{\tau^{\prime}}\right\| \geq\left\|v^{\tau}\right\|$. Then $R_{\tau^{\prime}}\left(\sum_{\tau \subseteq \eta} v^{\tau}\right)=0$. Using the bound on the norm of $\left\|R_{\tau^{\prime}} R_{\tau}\right\|$ established in step 2, this yields

$$
\begin{aligned}
& 0=\left\|R_{\tau^{\prime}}\left(\sum_{\tau \subseteq \eta} v^{\tau}\right)\right\| \geq\left\|v^{\tau^{\prime}}\right\|-\left\|\sum_{\tau \subseteq \eta, \tau \neq \tau^{\prime}} R_{\tau^{\prime}} v^{\tau}\right\| \geq \\
& \left\|v^{\tau^{\prime}}\right\|-\sum_{\tau \subseteq \eta, \tau \neq \tau^{\prime}}\left\|R_{\tau^{\prime}} R_{\tau} v^{\tau}\right\| \geq \\
& \left\|v^{\tau^{\prime}}\right\|-\left(C_{i}\right)^{2} \varepsilon\left\|v^{\tau^{\prime}}\right\|=\left\|v^{\tau^{\prime}}\right\|\left(1-\left(2^{i}-1\right)\left(C_{i}\right)^{2} \varepsilon\right) .
\end{aligned}
$$

Therefore, if $\varepsilon$ is chosen such that $\varepsilon<\frac{1}{\left(2^{i}-1\right)\left(C_{i}\right)^{2}}$, we get that $\left\|v^{\tau^{\prime}}\right\|=0$ and therefore $v^{\tau}=0$ for every $\tau \subseteq \eta$. This yields

$$
X_{\eta}=\bigoplus_{\tau \subseteq \eta} X^{\tau}
$$

as needed.

Remark 3.15. Note that in the above proof, the consistency assumption is crucial in the proof of Lemma 3.14 which in turn was crucial for step 1 of the above proof.

\section{Vanishing of cohomology}

Let $\Sigma$ be a pure $n$-dimensional infinite simplicial complex and let $G<A u t(\Sigma)$ be a closed subgroup. Assume that $(\Sigma, G)$ satisfies conditions $(\mathcal{B} 1)-(\mathcal{B} 4)$ defined in subsection 2.1 above. Assume further that all the 1-dimensional links of $\Sigma$ are compact. Fix a chamber $\triangle \in \Sigma(n)$. Let $\mu$ be the Haar measure on $G$. For $-1 \leq i \leq n$, denote $\triangle(i)$ to be the $i$-dimensional simplices of $\triangle$. For $\sigma \in(\triangle(n) \cup \triangle(n-1) \cup \triangle(n-2))$ define $k_{\sigma} \in C_{c}(G)$ as

$$
k_{\sigma}=\frac{\chi_{G_{\sigma}}}{\mu\left(G_{\sigma}\right)}
$$

where $\chi_{G_{\sigma}}$ is the indicator function on $G_{\sigma}$ (note that by our assumptions $G_{\sigma}$ is a compact group). Observe that

- For $\sigma, \tau \in(\triangle(n) \cup \triangle(n-1) \cup \triangle(n-2))$, if $\tau \subset \sigma$, then $k_{\tau} k_{\sigma}=k_{\tau}$.

- For any continuous representation $\pi$ of $G$ on a Banach space $X$ and any $\sigma \in(\triangle(n) \cup \triangle(n-1) \cup \triangle(n-2)), \pi\left(k_{\sigma}\right)$ is a projection on the $X^{\pi\left(G_{\sigma}\right)}$ (recall that $X^{\pi\left(G_{\sigma}\right)}$ is the subspace of vectors fixed by $G_{\sigma}$ ).

These observations yields that for any two $\sigma, \sigma^{\prime} \in \triangle(n-1)$ and any representation $\pi$ of $G$, we can define the cosine of the angle between $\pi\left(k_{\sigma}\right)$ and $\pi\left(k_{\sigma^{\prime}}\right)$ as in definition 2.10 above:

$$
\begin{aligned}
& \cos \left(\angle\left(\pi\left(k_{\sigma}\right), \pi\left(k_{\sigma^{\prime}}\right)\right)\right)= \\
& \max \left\{\left\|\pi\left(k_{\sigma}\right) \pi\left(k_{\sigma^{\prime}}\right)-\pi\left(k_{\sigma \cap \sigma^{\prime}}\right)\right\|,\left\|\pi\left(k_{\sigma^{\prime}}\right) \pi\left(k_{\sigma}\right)-\pi\left(k_{\sigma \cap \sigma^{\prime}}\right)\right\|\right\}= \\
& \max \left\{\left\|\pi\left(k_{\sigma} k_{\sigma^{\prime}}-k_{\sigma \cap \sigma^{\prime}}\right)\right\|,\left\|\pi\left(k_{\sigma^{\prime}} k_{\sigma}-k_{\sigma \cap \sigma^{\prime}}\right)\right\|\right\} .
\end{aligned}
$$


Therefore we are in the setting of Theorem 3.13. Applying Theorem 3.13 combined with Theorem 2.5 yields the following:

Theorem 4.1. Let $\Sigma$ be a pure $n$-dimensional infinite simplicial complex and let $G<\operatorname{Aut}(\Sigma)$ be a closed subgroup. Assume that $(\Sigma, G)$ satisfy conditions $(\mathcal{B} 1)-(\mathcal{B} 4)$ and that there is $l \in \mathbb{N}$ such that all the l-dimensional links of $\Sigma$ are compact. Then there are constants $\gamma=\gamma(n)>0, \beta=\beta(n)>1$ such that for every representation $\pi$ of $G$ on a Banach space, if

$$
\sup _{\sigma \in \triangle(n-1)}\left\|\pi\left(k_{\sigma}\right)\right\| \leq \beta, \sup _{\sigma, \sigma^{\prime} \in \triangle(n-1)} \cos \left(\angle\left(\pi\left(k_{\sigma}\right), \pi\left(k_{\sigma^{\prime}}\right)\right)\right) \leq \gamma,
$$

and the projections $\pi\left(k_{\sigma}\right)$ with $\sigma \in \triangle(n-1)$ are consistent, then

$$
H^{*}(G, \pi)=\bigoplus_{\eta \subseteq \triangle} \widetilde{H}^{*-1}\left(D_{\eta} ; X^{\eta}\right)
$$

and

$$
H^{i}(G, \pi)=0 \text { for } i=1, \ldots, l .
$$

Proof. Denote $P_{\sigma}=\pi\left(k_{\sigma}\right)$ for $\sigma \in \triangle(n-2) \cup \triangle(n-1) \cup \triangle(n)$. Let $\beta=\beta_{0}>1$ and $\gamma$ as in Theorem 3.13. The assumptions on $\pi$ grantee that the $P_{\sigma}$ 's fulfil the conditions of Theorem 3.13 .

Therefore for every $\eta \subseteq \triangle, X_{\eta}=\bigoplus_{\tau \subseteq \eta} X^{\tau}$. The vanishing of cohomology follows from Theorem 2.5.

Note that the constants $\gamma, \beta$ depend only on the dimension $n$ (and not on any other characteristics of $\Sigma$ ).

We will show that there are sufficient conditions that grantee the fulfilment of the conditions of the theorem above in a class of representations $\mathcal{F}_{0}\left(\overline{\mathcal{E}_{3}\left(\mathcal{E}_{2}\left(\mathcal{E}_{1}\left(r, C_{1}\right), \theta_{2}\right), C_{3}\right)}, G, s_{0}\right)$ defined in the introduction for suitable choices of $s_{0}>0$ and $r$. We start by recalling the following result from [17] that connects the Schatten norm of the projection operators to condition $\left(\mathcal{B}_{\delta, r}\right)$ defined above:

Lemma 4.2. 17][Corollary 4.20] Let $\Sigma$ be a pure $n$-dimensional infinite simplicial complex and let $G<A u t(\Sigma)$ be a closed subgroup. Assume that $(\Sigma, G)$ satisfy conditions $(\mathcal{B} 1)-(\mathcal{B} 4)$ and condition $\left(\mathcal{B}_{\delta, r}\right)$, then for every $\sigma, \sigma^{\prime} \in \triangle(n-1)$,

$$
\left\|\lambda\left(k_{\sigma} k_{\sigma^{\prime}}-k_{\sigma \cap \sigma^{\prime}}\right)\right\|_{S^{r}} \leq \delta
$$

where $\lambda \in B\left(L^{2}\left(G_{\sigma \cap \sigma^{\prime}}, \mu\right)\right)$ is the left regular representation.

Using the above lemma, we are able to deduce arbitrary small angles between all the projections $\pi\left(k_{\sigma}\right)$ and $\pi\left(\sigma^{\prime}\right)$ given the condition $\left(\mathcal{B}_{\delta, r^{\prime}}\right)$ is fulfilled:

Lemma 4.3. Let $\Sigma$ be a pure $n$-dimensional infinite simplicial complex and let $G<\operatorname{Aut}(\Sigma)$ be a closed subgroup. Assume that $(\Sigma, G)$ satisfy conditions $(\mathcal{B} 1)-(\mathcal{B} 4)$ and that the 1-dimensional links of $\Sigma$ are finite.

Let $r>2, C_{1} \geq 1,1 \geq \theta_{2}>0, C_{3} \geq 1$ be constants. For every $\gamma>0, s_{0} \geq 0$, $2 \leq r^{\prime}<r$, there is a $\delta>0$ such that if $(\Sigma, G)$ satisfies condition $\left(\mathcal{B}_{\delta, r^{\prime}}\right)$, then for every $\pi \in \mathcal{F}\left(\overline{\mathcal{E}_{3}\left(\mathcal{E}_{2}\left(\mathcal{E}_{1}\left(r, C_{1}\right), \theta_{2}\right), C_{3}\right)}, G, s_{0}\right)$,

$$
\sup _{\sigma, \sigma^{\prime} \in \triangle(n-1)} \cos \left(\angle\left(\pi\left(k_{\sigma}\right), \pi\left(k_{\sigma^{\prime}}\right)\right)\right) \leq \gamma .
$$


Proof. Fix $\pi \in \mathcal{F}\left(\overline{\mathcal{E}_{3}\left(\mathcal{E}_{2}\left(\mathcal{E}_{1}\left(r, C_{1}\right), \theta_{2}\right), C_{3}\right)}, G, s_{0}\right)$. Let $\sigma, \sigma^{\prime} \in \triangle(n-1)$ be any two different $(n-1)$-dimensional faces of $\triangle$ and assume without loss of generality that

$$
\cos \left(\angle\left(\pi\left(k_{\sigma}\right), \pi\left(k_{\sigma^{\prime}}\right)\right)\right)=\left\|\pi\left(k_{\sigma} k_{\sigma^{\prime}}-k_{\sigma \cap \sigma^{\prime}}\right)\right\| .
$$

By Proposition 2.27 we have that

$$
\left\|\pi\left(k_{\sigma} k_{\sigma^{\prime}}-k_{\sigma \cap \sigma^{\prime}}\right)\right\| \leq e^{2 s_{0}}\left\|\lambda\left(k_{\sigma} k_{\sigma^{\prime}}-k_{\sigma \cap \sigma^{\prime}}\right) \otimes i d_{X}\right\|_{B\left(L^{2}\left(G_{\sigma \cap \sigma^{\prime}} ; X\right)\right)} .
$$

Note that for any Banach space $X$, we have that

$$
\left\|\lambda\left(k_{\sigma} k_{\sigma^{\prime}}-k_{\sigma \cap \sigma^{\prime}}\right) \otimes i d_{X}\right\|_{B\left(L^{2}\left(G_{\sigma \cap \sigma^{\prime}} ; X\right)\right)} \leq\left\|\lambda\left(k_{\sigma} k_{\sigma^{\prime}}-k_{\sigma \cap \sigma^{\prime}}\right)\right\|_{B\left(L^{1}\left(G_{\sigma \cap \sigma^{\prime}}\right)\right)} \leq 2 .
$$

Assuming that $\delta \leq 1$ and applying Lemma 4.2 and Corollary 2.26 (with $L=2$ ) yields that

$$
\left\|\lambda\left(k_{\sigma} k_{\sigma^{\prime}}-k_{\sigma \cap \sigma^{\prime}}\right) \otimes i d_{X}\right\|_{B\left(L^{2}\left(G_{\sigma \cap \sigma^{\prime}} ; X\right)\right)} \leq C_{3} 2(C \delta)^{\theta_{2}},
$$

where $C=C\left(C_{1}, r, r^{\prime}\right)$ is the constant given in Corollary 2.26. Therefore, we have that for every $\pi \in \mathcal{F}\left(\overline{\mathcal{E}_{3}\left(\mathcal{E}_{2}\left(\mathcal{E}_{1}\left(r, C_{1}\right), \theta_{2}\right), C_{3}\right)}, G, s_{0}\right)$,

$$
\cos \left(\angle\left(\pi\left(k_{\sigma}\right), \pi\left(k_{\sigma^{\prime}}\right)\right)\right) \leq e^{2 s_{0}} C_{3} 2(C \delta)^{\theta_{2}},
$$

and choosing $\delta=\frac{1}{C}\left(\frac{\gamma}{2 e^{2 s_{0}} C_{3}}\right)^{\frac{1}{\theta_{2}}}$ yields the needed inequality.

The implication of the above lemma is that when applying Theorem 4.1 on a class of representations of the form $\mathcal{F}\left(\overline{\mathcal{E}_{3}\left(\mathcal{E}_{2}\left(\mathcal{E}_{1}\left(r, C_{1}\right), \theta_{2}\right), C_{3}\right)}, G, s_{0}\right)$, one can replace the condition

$$
\sup _{\sigma, \sigma^{\prime} \in \triangle(n-1)} \cos \left(\angle\left(\pi\left(k_{\sigma}\right), \pi\left(k_{\sigma^{\prime}}\right)\right)\right) \leq \gamma,
$$

by the condition $\left(\mathcal{B}_{\delta, r^{\prime}}\right)$ for suitable values of $\delta$ and $r^{\prime}$ :

Theorem 4.4. Let $\Sigma$ be a pure $n$-dimensional infinite simplicial complex and let $G<\operatorname{Aut}(\Sigma)$ be a closed subgroup. Assume that $(\Sigma, G)$ satisfy conditions $(\mathcal{B} 1)-(\mathcal{B} 4)$ and that there is a $l \geq 1$ such that all the l-dimensional links of $\Sigma$ are compact.

Let $r>r^{\prime} \geq 2, C_{1} \geq 1,1 \geq \theta_{2}>0, C_{3} \geq 1$ be constants. Then there are $s_{0}=s_{0}(n)>0$ and

$$
\delta=\delta\left(n, r, r^{\prime}, C_{1}, \theta_{2}, C_{3}\right)>0
$$

such that if $(\Sigma, G)$ fulfil condition $\left(\mathcal{B}_{\delta, r^{\prime}}\right)$ and if the projections $\pi\left(k_{\sigma}\right)$ with $\sigma \in$ $\triangle(n-1)$ are consistent, then

$$
H^{*}(G, \pi)=\bigoplus_{\eta \subseteq \triangle} \widetilde{H}^{*-1}\left(D_{\eta} ; X^{\eta}\right)
$$

and

$$
H^{i}(G, \pi)=0 \text { for } i=1, \ldots, l,
$$

for every $\pi \in \mathcal{F}\left(\overline{\mathcal{E}_{3}\left(\mathcal{E}_{2}\left(\mathcal{E}_{1}\left(r, C_{1}\right), \theta_{2}\right), C_{3}\right)}, G, s_{0}\right)$. 
Proof. Let $\beta>1, \gamma>0$ be the constants given by Theorem 4.1

Choose $s_{0}=\ln (\beta)$, by this choice the inequality

$$
\max _{\sigma \in \triangle(n-1)}\left\|\pi\left(k_{\sigma}\right)\right\| \leq \sup _{g \in \bigcup_{\sigma \in \Delta(n-1)} G_{\sigma}}\|\pi(g)\| \leq \sup _{g \in \bigcup_{\tau \in \Delta(n-2)} G_{\tau}}\|\pi(g)\| \leq e^{s_{0}}=\beta
$$

is satisfied for each $\pi \in \mathcal{F}\left(\overline{\mathcal{E}_{3}\left(\mathcal{E}_{2}\left(\mathcal{E}_{1}\left(r, C_{1}\right), \theta_{2}\right), C_{3}\right)}, G, s_{0}\right)$.

By Lemma 4.3. we can choose $\delta>0$ small enough such that the condition $\left(\mathcal{B}_{\delta, r^{\prime}}\right)$ we imply that

$$
\sup _{\sigma, \sigma^{\prime} \in \triangle(n-1)} \cos \left(\angle\left(\pi\left(k_{\sigma}\right), \pi\left(k_{\sigma^{\prime}}\right)\right)\right) \leq \gamma
$$

for every $\pi \in \mathcal{F}\left(\overline{\mathcal{E}_{3}\left(\mathcal{E}_{2}\left(\mathcal{E}_{1}\left(r, C_{1}\right), \theta_{2}\right), C_{3}\right)}, G, s_{0}\right)$.

Therefore for this choice of $s_{0}>0$ and $\delta>0$, the conditions of Theorem 4.1 are fulfilled and the conclusion follows.

The unsatisfactory part of the above theorem is the assumption of consistency of the projections $\pi\left(k_{\sigma}\right)$. We will show that when passing to the class $\mathcal{F}_{0}\left(\overline{\mathcal{E}_{3}\left(\mathcal{E}_{2}\left(\mathcal{E}_{1}\left(r, C_{1}\right), \theta_{2}\right), C_{3}\right)}, G, s_{0}\right)$ (in which the dual representations are continuous) this always assumption holds.

Lemma 4.5. Let $\Sigma$ be a pure $n$-dimensional infinite simplicial complex and let $G<\operatorname{Aut}(\Sigma)$ be a closed subgroup. Assume that $(\Sigma, G)$ satisfy conditions $(\mathcal{B} 1)-(\mathcal{B} 4)$.

Let $\pi$ be a continuous representation on a Banach space $X$ such that

$$
\sup _{\sigma \in \triangle(n-1)}\left\|\pi\left(k_{\sigma}\right)\right\| \leq \beta_{0}, \sup _{\sigma, \sigma^{\prime} \in \triangle(n-1)} \cos \left(\angle\left(\pi\left(k_{\sigma}\right), \pi\left(k_{\sigma^{\prime}}\right)\right)\right) \leq \gamma_{0},
$$

where $\beta_{0}>1, \gamma_{0}>0$ are the constants given by Corollary 2.14.

If the dual representation $\pi^{*}$ is continuous, then the projections $\pi\left(k_{\sigma}\right)$ for $\sigma \in \triangle(n-1)$ are consistent.

Proof. Let $\mathcal{F}=\left\{\pi, \pi^{*}\right\}$. Note that for $\pi^{*}$ the following holds:

$$
\sup _{\sigma \in \triangle(n-1)}\left\|\pi^{*}\left(k_{\sigma}\right)\right\| \leq \beta_{0}, \sup _{\sigma, \sigma^{\prime} \in \triangle(n-1)} \cos \left(\angle\left(\pi^{*}\left(k_{\sigma}\right), \pi^{*}\left(k_{\sigma^{\prime}}\right)\right)\right) \leq \gamma_{0} .
$$

Therefore by Corollary 2.14 for every $\tau \varsubsetneqq \triangle$,

$$
\left(\frac{1}{n+1-|\tau|} \sum_{\sigma \in \triangle(n-1), \tau \subseteq \sigma} k_{\sigma}\right)^{i} \stackrel{i \rightarrow \infty}{\longrightarrow} k_{\tau},
$$

where the convergence is in $C_{\mathcal{F}}$ and $\pi\left(k_{\tau}\right)$ and $\pi^{*}\left(k_{\tau}\right)$ are projections on $X^{\pi\left(G_{\tau}\right)}$ and $\left(X^{*}\right)^{\pi^{*}\left(G_{\tau}\right)}$ respectively.

By Proposition 3.8, in order to prove consistency, it is enough to show that

$$
\forall \tau \varsubsetneqq \triangle, \forall \sigma \in \triangle(n-1), \tau \subseteq \sigma \Rightarrow \pi\left(k_{\tau}\right) \pi\left(k_{\sigma}\right)=\pi\left(k_{\tau}\right) .
$$

We will prove the following condition which is actually stronger:

$$
\forall \tau \varsubsetneqq \triangle, \forall g \in G_{\tau}, \pi\left(k_{\tau}\right) \pi(g)=\pi\left(k_{\tau}\right) .
$$


Fix some $\tau \varsubsetneqq \triangle$ and $g \in G_{\tau}$. For every $v \in X, w \in X^{*}$ we have that

$$
\begin{aligned}
\left\langle\pi\left(k_{\tau}\right) \pi(g) \cdot v, w\right\rangle & =\left\langle v, \pi^{*}(g) \pi^{*}\left(k_{\tau}\right) \cdot w\right\rangle \\
& =\left\langle v, \pi^{*}\left(k_{\tau}\right) \cdot w\right\rangle \\
& =\left\langle\pi\left(k_{\tau}\right) \cdot v, w\right\rangle .
\end{aligned}
$$

Therefore, $\pi\left(k_{\tau}\right) \pi(g)=\pi\left(k_{\tau}\right)$ as needed.

Remark 4.6. We note that if $G$ is a discrete group, then the condition of $\pi^{*}$ being continuous always holds (since it is vacuous).

As a corollary of the Lemma 4.5 we deduce the following theorem:

Theorem 4.7. Let $\Sigma$ be a pure $n$-dimensional infinite simplicial complex and let $G<\operatorname{Aut}(\Sigma)$ be a closed subgroup. Assume that $(\Sigma, G)$ satisfy conditions $(\mathcal{B} 1)-(\mathcal{B} 4)$ and that there is $l \in \mathbb{N}$ such that all the l-dimensional links of $\Sigma$ are compact.

Let $r>r^{\prime} \geq 2, C_{1} \geq 1,1 \geq \theta_{2}>0, C_{3} \geq 1$ be constants. Then there are $s_{0}=s_{0}(n)>0$ and

$$
\delta=\delta\left(n, r, r^{\prime}, C_{1}, \theta_{2}, C_{3}\right)>0
$$

such that if $(\Sigma, G)$ fulfil condition $\left(\mathcal{B}_{\delta, r^{\prime}}\right)$, then

$$
H^{*}(G, \pi)=\bigoplus_{\eta \subseteq \triangle} \widetilde{H}^{*-1}\left(D_{\eta} ; X^{\eta}\right),
$$

and

$$
H^{i}(G, \pi)=0 \text { for } i=1, \ldots, l,
$$

for every $\pi \in \mathcal{F}_{0}\left(\overline{\mathcal{E}_{3}\left(\mathcal{E}_{2}\left(\mathcal{E}_{1}\left(r, C_{1}\right), \theta_{2}\right), C_{3}\right)}, G, s_{0}\right)$.

Proof. By Lemma 4.5, the projections $\pi\left(k_{\sigma}\right)$ with $\sigma \in \triangle(n-1)$ are consistent for every $\pi \in \mathcal{F}_{0}\left(\overline{\mathcal{E}_{3}\left(\mathcal{E}_{2}\left(\mathcal{E}_{1}\left(r, C_{1}\right), \theta_{2}\right), C_{3}\right)}, G, s_{0}\right)$ and therefore we can apply Theorem 4.4.

We recall that by Theorem 2.29 stated above, for a continuous representation $\pi$ on a Banach space $X$, if $X$ is an Asplund space then $\pi^{*}$ is continuous. We also recall that all reflexive Banach spaces are Asplund spaces and it was shown in the introduction that the subclass of reflexive Banach spaces of $\overline{\mathcal{E}_{3}\left(\mathcal{E}_{2}\left(\mathcal{E}_{1}\left(r, C_{1}\right), \theta_{2}\right), C_{3}\right)}$ contains several interesting families of Banach spaces.

As stated in subsection 2.1 above, the main example of couples $(\Sigma, G)$ satisfying the conditions $(\mathcal{B} 1)-(\mathcal{B} 4)$ are groups $G$ with a BN-pair acting on a building $\Sigma$. In Proposition 2.9 we showed that the condition $\left(\mathcal{B}_{\delta, r}\right)$ can also be deduced for these examples for suitable values of $r$. Therefore we can deduce the following corollary:

Corollary 4.8. Let $G$ be a group coming from a BN-pair and let $\Sigma$ be the $n$ dimensional building on which it acts. Assume that $n>1$ and there is some $l \geq 1$ such that all the $l$-dimensional links of $\Sigma$ are compact. Denote by $q$ the thickness of the building $\Sigma$ and let $m^{\prime}$ be the smallest integer such that all the links of 1-dimensional links of $\Sigma$ are generalized $m$-gons with $m \leq m^{\prime}$. 
Let

$$
r> \begin{cases}4 & m^{\prime}=3 \\ 8 & m^{\prime}=4 \\ 18 & m^{\prime}=6 \\ 20 & m^{\prime}=8\end{cases}
$$

and $C_{1} \geq 1,1 \geq \theta_{2}>0, C_{3} \geq 1$ be constants, then there are $s_{0}=s_{0}(n)>0$ and

$$
Q=Q\left(n, r, m^{\prime}, C_{1}, \theta_{2}, C_{3}, s_{0}\right) \in \mathbb{N}
$$

such that if $q \geq Q$, then

$$
H^{*}(G, \pi)=\bigoplus_{\eta \subseteq \triangle} \widetilde{H}^{*-1}\left(D_{\eta} ; X^{\eta}\right)
$$

and

$H^{i}(G, \pi)=0$ for $i=1, \ldots, l$,

for every $\pi \in \mathcal{F}_{0}\left(\overline{\mathcal{E}_{3}\left(\mathcal{E}_{2}\left(\mathcal{E}_{1}\left(r, C_{1}\right), \theta_{2}\right), C_{3}\right)}, G, s_{0}\right)$.

Proof. Fix

$$
r^{\prime}= \begin{cases}\frac{4+r}{2} & m^{\prime}=3 \\ \frac{8+r}{2} & m^{\prime}=4 \\ \frac{18+r}{2} & m^{\prime}=6 \\ \frac{20+r}{2} & m^{\prime}=8\end{cases}
$$

and let $s_{0}(n)>0, \delta=\delta\left(n, r, r^{\prime}, C_{1}, \theta_{2}, C_{3}, s_{0}\right)>0$ be as in Theorem 4.7 By Proposition 2.9. there is a large enough $Q$ such that for every $q \geq Q, \Sigma$ fulfils the condition $\left(\mathcal{B}_{\delta, r^{\prime}}\right)$ and we are done by Theorem 4.7 .

\section{A Angle between projections without the con- sistency assumption}

Under the notations of definition 2.10, given $\tau, \tau^{\prime} \subseteq \triangle$ such that $P_{\tau}, P_{\tau^{\prime}}, P_{\tau \cap \tau^{\prime}}$ exist, we can define $\cos \left(\angle\left(P_{\tau}, P_{\tau^{\prime}}\right)\right)$ as

$$
\cos \left(\angle\left(P_{\tau}, P_{\tau^{\prime}}\right)\right)=\max \left\{\left\|P_{\tau} P_{\tau^{\prime}}\left(I-P_{\tau \cap \tau^{\prime}}\right)\right\|,\left\|P_{\tau^{\prime}} P_{\tau}\left(I-P_{\tau \cap \tau^{\prime}}\right)\right\|\right\} .
$$

We note that in this definition, we do not assume that $P_{\tau \cap \tau^{\prime}} P_{\tau}=P_{\tau \cap \tau^{\prime}}$ or that $P_{\tau \cap \tau^{\prime}} P_{\tau^{\prime}}=P_{\tau \cap \tau^{\prime}}$. However, even without this assumption of consistency, we can derive a theorem similar to Theorem 3.10 ,

Theorem A.1. Let $X, \triangle$ and $P_{\sigma} \in \triangle(n-1) \cup \triangle(n)$ be as in definition 3.1 above. Assume that for every $\eta \in \triangle(n-2)$, the projection $P_{\eta}$ exists and for $\sigma \in \triangle(n-1)$, if $\eta \subset \sigma$ then $P_{\eta} P_{\sigma}=P_{\eta}$.

Then there is $\beta>1$ such that for every $\varepsilon>0$ there is $\gamma>0$ such that if

$$
\max _{\sigma \in \triangle(n-1)}\left\{\left\|P_{\sigma}\right\| \leq \beta \text { and } \max \left\{\cos \left(\angle\left(P_{\sigma}, P_{\sigma^{\prime}}\right)\right): \sigma, \sigma^{\prime} \in \triangle(n-1)\right\} \leq \gamma,\right.
$$

then $P_{\tau}$ exist for any $\tau \subseteq \triangle$ and for every $\tau, \tau^{\prime} \subseteq \triangle$,

$$
\cos \left(L\left(P_{\tau}, P_{\tau^{\prime}}\right)\right) \leq \varepsilon
$$


We will start with the following lemma asserting that under the assumptions of the above theorems the projections are bounded and "almost" commute:

Lemma A.2. Let $X, \triangle$ and $P_{\sigma} \in \triangle(n-1) \cup \triangle(n)$ be as in definition 3.1 above. Assume that for every $\eta \in \triangle(n-2)$, the projection $P_{\eta}$ exists and for $\sigma \in \triangle(n-1)$, if $\eta \subset \sigma$ then $P_{\eta} P_{\sigma}=P_{\eta}$.

Then there is $\beta>1$ such that for every $\varepsilon>0$ there is $\gamma>0$ such that if

$$
\max _{\sigma \in \triangle(n-1)}\left\{\left\|P_{\sigma}\right\| \leq \beta \text { and } \max \left\{\cos \left(\angle\left(P_{\sigma}, P_{\sigma^{\prime}}\right)\right): \sigma, \sigma^{\prime} \in \triangle(n-1)\right\} \leq \gamma .\right.
$$

then the following holds:

1. For every $\tau \subseteq \triangle, P_{\tau}$ exists and $\left\|P_{\tau}\right\| \leq 4(n+1)+2$.

2. For every $\tau, \tau^{\prime} \subseteq \triangle,\left\|P_{\tau} P_{\tau^{\prime}}-P_{\tau^{\prime}} P_{\tau}\right\| \leq \varepsilon$.

Proof. Take $\beta=\min \left\{\beta_{0}, \frac{2(n+1)+1}{2(n+1)}\right\}$ and $\gamma \leq \gamma_{0}$. The proof of the first assertion is identical to the one given in the proof of Theorem 3.10 above (note that the consistency assumption was not used in this proof).

We are left with proving the second assertion. Fix $\varepsilon>0$ and assume that $\gamma \leq \gamma_{0}$. Then for every $\tau, \tau^{\prime} \subseteq \triangle$ and every $i \in \mathbb{N}$ the following holds:

$$
\begin{aligned}
& \left\|P_{\tau} P_{\tau^{\prime}}-T_{\tau}^{i} T_{\tau^{\prime}}^{i}\right\| \leq\left\|P_{\tau}\left(P_{\tau^{\prime}}-T_{\tau^{\prime}}^{i}\right)\right\|+\left\|\left(P_{\tau}-T_{\tau}^{i}\right) T_{\tau^{\prime}}^{i}\right\| \leq \\
& \quad\left\|P_{\tau}\right\|\left\|P_{\tau^{\prime}}-T_{\tau^{\prime}}^{i}\right\|+\left\|P_{\tau}-T_{\tau}^{i}\right\|\left\|T_{\tau^{\prime}}\right\|^{i} .
\end{aligned}
$$

Note that $\left\|T_{\tau^{\prime}}\right\| \leq \beta \leq \frac{2(n+1)+1}{2(n+1)}$ and that $\left\|P_{\tau}\right\| \leq 4(n+1)+2$. Combining these bounds with Corollary 2.14 yields

$$
\begin{aligned}
& \left\|P_{\tau} P_{\tau^{\prime}}-T_{\tau}^{i} T_{\tau^{\prime}}^{i}\right\| \leq(4(n+1)+2) 4(n+1)\left(\frac{2(n+1)-1}{2(n+1)}\right)^{i-1} \\
& +(4(n+1)+2)\left(\frac{4(n+1)^{2}-1}{4(n+1)^{2}}\right)^{i-1} .
\end{aligned}
$$

The right-hand side of the above inequality goes to 0 as $i$ tends to $\infty$ and therefore we can choose $i_{0}$ such that $\left\|P_{\tau} P_{\tau^{\prime}}-T_{\tau}^{i_{0}} T_{\tau^{\prime}}^{i_{0}}\right\| \leq \frac{\varepsilon}{4}$ (note that this choice of $i_{0}$ holds for every $\gamma \leq \gamma_{0}$ ). Similarly, $\left\|P_{\tau^{\prime}} P_{\tau}-T_{\tau^{\prime}}^{i_{0}} T_{\tau}^{i_{0}}\right\| \leq \frac{\varepsilon}{4}$ and therefore

$$
\left\|P_{\tau} P_{\tau^{\prime}}-P_{\tau^{\prime}} P_{\tau}\right\| \leq \frac{\varepsilon}{2}+\left\|T_{\tau}^{i_{0}} T_{\tau^{\prime}}^{i_{0}}-T_{\tau^{\prime}}^{i_{0}} T_{\tau}^{i_{0}}\right\| .
$$

We are left to prove that by choosing $\gamma$ small enough, we can assure that

$$
\left\|T_{\tau}^{i_{0}} T_{\tau^{\prime}}^{i_{0}}-T_{\tau^{\prime}}^{i_{0}} T_{\tau}^{i_{0}}\right\| \leq \frac{\varepsilon}{2}
$$

when $i_{0}$ is fixed. As in the proof of Theorem 3.5. we note that for any $\sigma, \sigma^{\prime} \in$ $\triangle(n-1)$,

$$
\left\|P_{\sigma} P_{\sigma^{\prime}}-P_{\sigma^{\prime}} P_{\sigma}\right\| \leq 2 \gamma .
$$

Therefore $\left\|T_{\tau} T_{\tau^{\prime}}-T_{\tau^{\prime}} T_{\tau}\right\| \leq 2 \gamma$. By permuting pairwise $T_{\tau}$ and $T_{\tau^{\prime}}$ we get that

$$
\left\|T_{\tau}^{i_{0}} T_{\tau^{\prime}}^{i_{0}}-T_{\tau^{\prime}}^{i_{0}} T_{\tau}^{i_{0}}\right\| \leq 2 i_{0}^{2}\left\|T_{\tau}\right\|^{i_{0}-1}\left\|T_{\tau^{\prime}}\right\|^{i_{0}-1} \gamma \leq 2 i_{0}^{2}\left(\frac{2(n+1)+1}{2(n+1)}\right)^{i_{0}} \gamma .
$$


Recall that $i_{0}$ is fixed and therefore we can choose $\gamma$ small enough such that

$$
\left\|T_{\tau}^{i_{0}} T_{\tau^{\prime}}^{i_{0}}-T_{\tau^{\prime}}^{i_{0}} T_{\tau}^{i_{0}}\right\| \leq \frac{\varepsilon}{2}
$$

and we are done.

After this, we are ready to prove Theorem A.1 above:

Proof. Let $\beta$ be as in Lemma A.2 (note that $\beta \leq \beta_{0}$ ). Fix $\varepsilon>0$ and let $\varepsilon_{1}>0$, $\varepsilon_{2}>0$ be constants that will be determined later. Let $\gamma_{1}$ be the bound of the cosine of the angles of Theorem 3.5 that correspond to $\varepsilon_{1}$. Similarly, let $\gamma_{2}$ be the bound of the cosine of the angles of Lemma A.2 that correspond to $\varepsilon_{2}$. Choose $\gamma=\min \left\{\gamma_{1}, \gamma_{2}\right\}$.

Let $\tau, \tau^{\prime} \subseteq \triangle$. Without loss of generality, it is sufficient to show that

$$
\left\|P_{\tau} P_{\tau^{\prime}}\left(I-P_{\tau \cap \tau^{\prime}}\right)\right\| \leq \varepsilon .
$$

Note that by the same arguments of the proof of Theorem 3.10, we can assume that $\tau \cap \tau^{\prime} \in \triangle(n-1-k)$ with $0 \leq k \leq n$.

Let $\sigma_{0}, \ldots, \sigma_{k} \in \triangle(n-1)$ be the pairwise disjoint simplices that contain $\tau \cap \tau^{\prime}$. Without loss of generality we can assume that

$$
\tau \subseteq \sigma_{0}, \ldots, \tau \subseteq \sigma_{j} \text { and } \tau^{\prime} \subseteq \sigma_{j+1}, \ldots, \tau^{\prime} \subseteq \sigma_{k} .
$$

We note that

$$
P_{\tau}=P_{\sigma_{0}} \ldots P_{\sigma_{j}} P_{\tau},
$$

and

Therefore

$$
P_{\tau^{\prime}}=P_{\sigma_{j+1}} \ldots P_{\sigma_{k}} P_{\tau^{\prime}}
$$

$$
P_{\tau} P_{\tau^{\prime}}\left(I-P_{\tau \cap \tau^{\prime}}\right)=P_{\sigma_{0}} \ldots P_{\sigma_{j}} P_{\tau} P_{\sigma_{j+1}} \ldots P_{\sigma_{k}} P_{\tau^{\prime}}\left(I-P_{\tau \cap \tau^{\prime}}\right) .
$$

We note that

$$
\begin{aligned}
& \left\|P_{\sigma_{0}} \ldots P_{\sigma_{j}} P_{\tau} P_{\sigma_{j+1}} \ldots P_{\sigma_{k}} P_{\tau^{\prime}}\left(I-P_{\tau \cap \tau^{\prime}}\right)\right\| \leq \\
& \quad\left\|P_{\sigma_{0}} \ldots P_{\sigma_{j-1}}\left(P_{\sigma_{j}} P_{\tau}-P_{\tau} P_{\sigma_{j}}\right) P_{\sigma_{j+1}} \ldots P_{\sigma_{k}} P_{\tau^{\prime}}\left(I-P_{\tau \cap \tau^{\prime}}\right)\right\|+ \\
& \quad\left\|P_{\sigma_{0}} \ldots P_{\sigma_{j-1}} P_{\tau} P_{\sigma_{j}} P_{\sigma_{j+1}} \ldots P_{\sigma_{k}} P_{\tau^{\prime}}\left(I-P_{\tau \cap \tau^{\prime}}\right)\right\| \leq \\
& \quad(4(n+1)+2)^{k+1}(4(n+1)+3) \varepsilon_{2}+ \\
& \left\|P_{\sigma_{0}} \ldots P_{\sigma_{j-1}} P_{\tau} P_{\sigma_{j}} P_{\sigma_{j+1}} \ldots P_{\sigma_{k}} P_{\tau^{\prime}}\left(I-P_{\tau \cap \tau^{\prime}}\right)\right\|,
\end{aligned}
$$

where the last inequality is due to Lemma A.2 Applying the same argument several times, we get that

$$
\begin{aligned}
& \left\|P_{\tau} P_{\tau^{\prime}}\left(I-P_{\tau \cap \tau^{\prime}}\right)\right\|=\left\|P_{\sigma_{0}} \ldots P_{\sigma_{j}} P_{\tau} P_{\sigma_{j+1}} \ldots P_{\sigma_{k}} P_{\tau^{\prime}}\left(I-P_{\tau \cap \tau^{\prime}}\right)\right\| \leq \\
& (j+2)(4(n+1)+2)^{k+1}(4(n+1)+3) \varepsilon_{2}+\left\|P_{\tau} P_{\sigma_{0}} \ldots P_{\sigma_{k}}\left(I-P_{\tau \cap \tau^{\prime}}\right) P_{\tau^{\prime}}\right\| \leq \\
& (j+2)(4(n+1)+2)^{k+1}(4(n+1)+3) \varepsilon_{2}+(4(n+1)+2)^{2} \cos \left(L\left(P_{\sigma_{0}}, \ldots, P_{\sigma_{k}}\right)\right) \leq \\
& (j+2)(4(n+1)+2)^{k+1}(4(n+1)+3) \varepsilon_{2}+(4(n+1)+2)^{2} \varepsilon_{1} \leq \\
& (n+2)(4(n+1)+2)^{n+1}(4(n+1)+3) \varepsilon_{2}+(4(n+1)+2)^{2} \varepsilon_{1}
\end{aligned}
$$

Therefore choosing

$$
\varepsilon_{1}=\frac{\varepsilon}{2(4(n+1)+2)^{2}}, \varepsilon_{2}=\frac{\varepsilon}{2(n+2)(4(n+1)+2)^{n+1}(4(n+1)+3)},
$$

yields the needed inequality. 


\section{References}

[1] Peter Abramenko and Kenneth S. Brown. Buildings, volume 248 of Graduate Texts in Mathematics. Springer, New York, 2008. Theory and applications.

[2] W. Ballmann and J. Świątkowski. On $L^{2}$-cohomology and property (T) for automorphism groups of polyhedral cell complexes. Geom. Funct. Anal., 7(4):615-645, 1997.

[3] Bachir Bekka, Pierre de la Harpe, and Alain Valette. Kazhdan's property (T), volume 11 of New Mathematical Monographs. Cambridge University Press, Cambridge, 2008.

[4] Yoav Benyamini and Joram Lindenstrauss. Geometric nonlinear functional analysis. Vol. 1, volume 48 of American Mathematical Society Colloquium Publications. American Mathematical Society, Providence, RI, 2000.

[5] Jöran Bergh and Jörgen Löfström. Interpolation spaces. An introduction. Springer-Verlag, Berlin-New York, 1976. Grundlehren der Mathematischen Wissenschaften, No. 223.

[6] A. Borel and N. Wallach. Continuous cohomology, discrete subgroups, and representations of reductive groups, volume 67 of Mathematical Surveys and Monographs. American Mathematical Society, Providence, RI, second edition, 2000.

[7] Tim de Laat and Mikael de la Salle. Approximation properties for noncommutative $l^{p}$-spaces of high rank lattices and nonembeddability of expanders. http://arxiv.org/abs/1403.6415, 2014.

[8] Jan Dymara and Tadeusz Januszkiewicz. Cohomology of buildings and their automorphism groups. Invent. Math., 150(3):579-627, 2002.

[9] Mikhail Ershov and Andrei Jaikin-Zapirain. Property (T) for noncommutative universal lattices. Invent. Math., 179(2):303-347, 2010.

[10] Walter Feit and Graham Higman. The nonexistence of certain generalized polygons. J. Algebra, 1:114-131, 1964.

[11] Robert C. James. Uniformly non-square Banach spaces. Ann. of Math. (2), 80:542-550, 1964.

[12] Fritz John. Extremum problems with inequalities as subsidiary conditions. In Studies and Essays Presented to R. Courant on his 60th Birthday, January 8, 1948, pages 187-204. Interscience Publishers, Inc., New York, N. Y., 1948.

[13] Martin Kassabov. Subspace arrangements and property T. Groups Geom. Dyn., 5(2):445-477, 2011.

[14] Michael G. Megrelishvili. Fragmentability and continuity of semigroup actions. Semigroup Forum, 57(1):101-126, 1998. 
[15] V. D. Mil' man and H. Wolfson. Minkowski spaces with extremal distance from the Euclidean space. Israel J. Math., 29(2-3):113-131, 1978.

[16] Piotr W. Nowak. Group actions on Banach spaces. In Handbook of group actions. Vol. II, volume 32 of Adv. Lect. Math. (ALM), pages 121-149. Int. Press, Somerville, MA, 2015.

[17] Izhar Oppenheim. Averaged projections, angles between groups and strengthening of property (T). Mathematische Annalen, To appear., 2016.

[18] Albrecht Pietsch. History of Banach spaces and linear operators. Birkhäuser Boston, Inc., Boston, MA, 2007.

[19] G. Pisier. Some applications of the complex interpolation method to Banach lattices. J. Analyse Math., 35:264-281, 1979.

[20] Gilles Pisier and Quan Hua Xu. Random series in the real interpolation spaces between the spaces $v_{p}$. In Geometrical aspects of functional analysis (1985/86), volume 1267 of Lecture Notes in Math., pages 185-209. Springer, Berlin, 1987.

[21] Gilles Pisier and Quanhua Xu. Non-commutative $L^{p}$-spaces. In Handbook of the geometry of Banach spaces, Vol. 2, pages 1459-1517. North-Holland, Amsterdam, 2003.

[22] Mikael de la Salle. Towards strong Banach property (T) for $\mathrm{SL}(3, \mathbb{R})$. Israel J. Math., 211(1):105-145, 2016.

[23] Yehuda Shalom. The algebraization of Kazhdan's property (T). In International Congress of Mathematicians. Vol. II, pages 1283-1310. Eur. Math. Soc., Zürich, 2006.

[24] Nicole Tomczak-Jaegermann. Computing 2-summing norm with few vectors. Ark. Mat., 17(2):273-277, 1979.

[25] Hendrik Van Maldeghem. Generalized polygons. Modern Birkhäuser Classics. Birkhäuser/Springer Basel AG, Basel, 1998. [2011 reprint of the 1998 original] [MR1725957].

[26] D. Yost. Asplund spaces for beginners. Acta Univ. Carolin. Math. Phys., 34(2):159-177, 1993. Selected papers from the 21st Winter School on Abstract Analysis (Poděbrady, 1993).

[27] Andrzej Żuk. La propriété (T) de Kazhdan pour les groupes agissant sur les polyèdres. C. R. Acad. Sci. Paris Sér. I Math., 323(5):453-458, 1996. 